\title{
Invisible trophic links? Quantifying the importance of non-standard food sources for key intertidal avian predators in the Eastern Atlantic
}

\author{
Pedro M. Lourenço*, Teresa Catry, Ricardo J. Lopes, Theunis Piersma, José P. Granadeiro \\ *Corresponding author: p.m.g.lourenco@gmail.com
}

Marine Ecology Progress Series 563: 219-232 (2017)

Figure S1: Sensitivity analysis on the effects of changing isotope trophic discrimination factors in estimates of the relative contribution of different food sources to the diet of shorebirds in the four study areas. Colours (and the z-axis) in each graphic indicate the proportion contribution of each potential food sources, as estimated by SIAR mixing models, with varying values of trophic discrimination for nitrogen (y-axis; 2.4 to $4.4 \% \circ \delta^{15} \mathrm{~N}$ ) and carbon (x-axis; -0.9 to $1.7 \% \circ \delta^{13} \mathrm{C}$ ). Each page presents a different shorebird species/site. Taxa included in each food source group are listed in Table $\mathrm{S} 1$.

\section{Ruddy turnstone (Arenaria interpres), Tejo estuary}
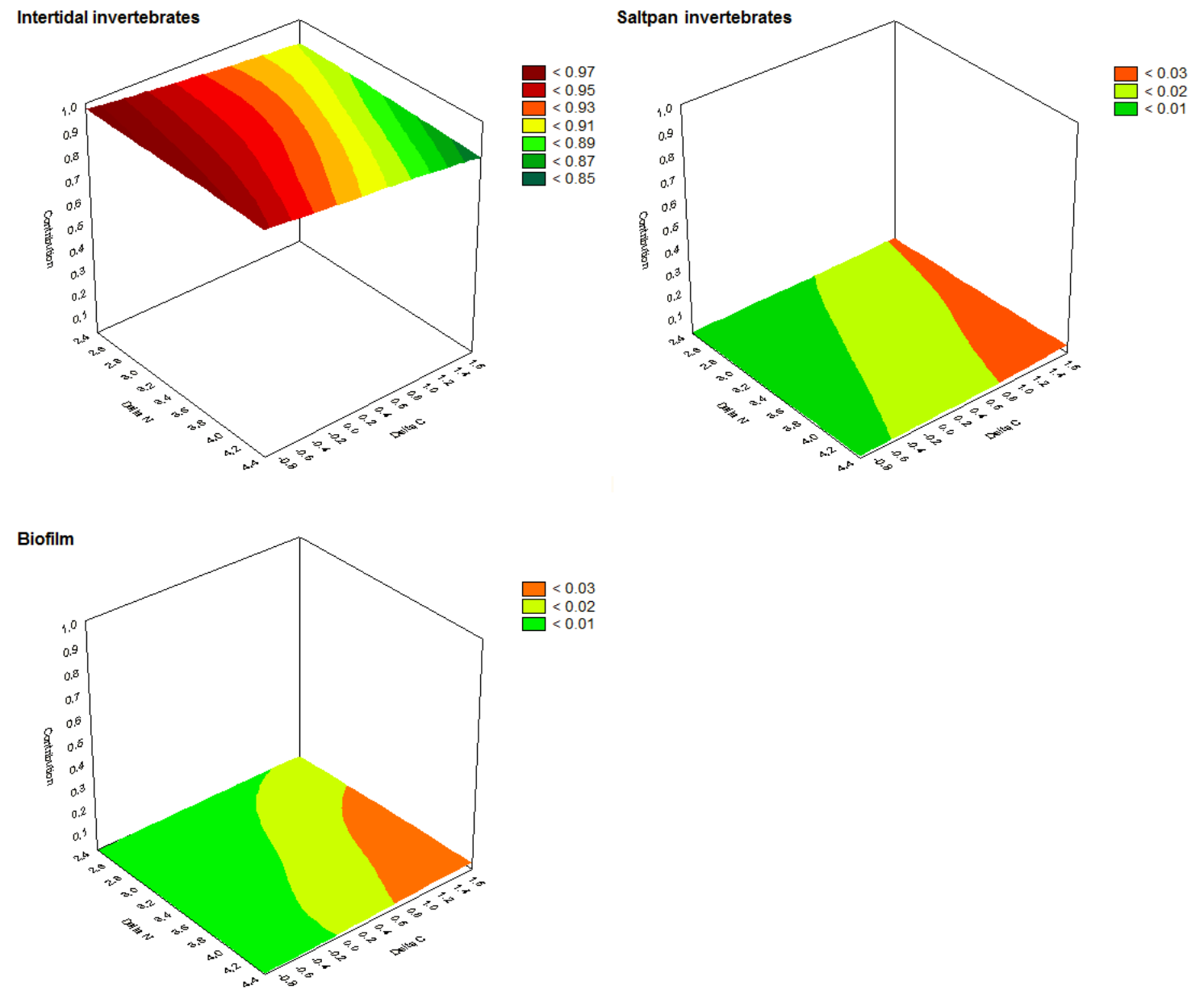

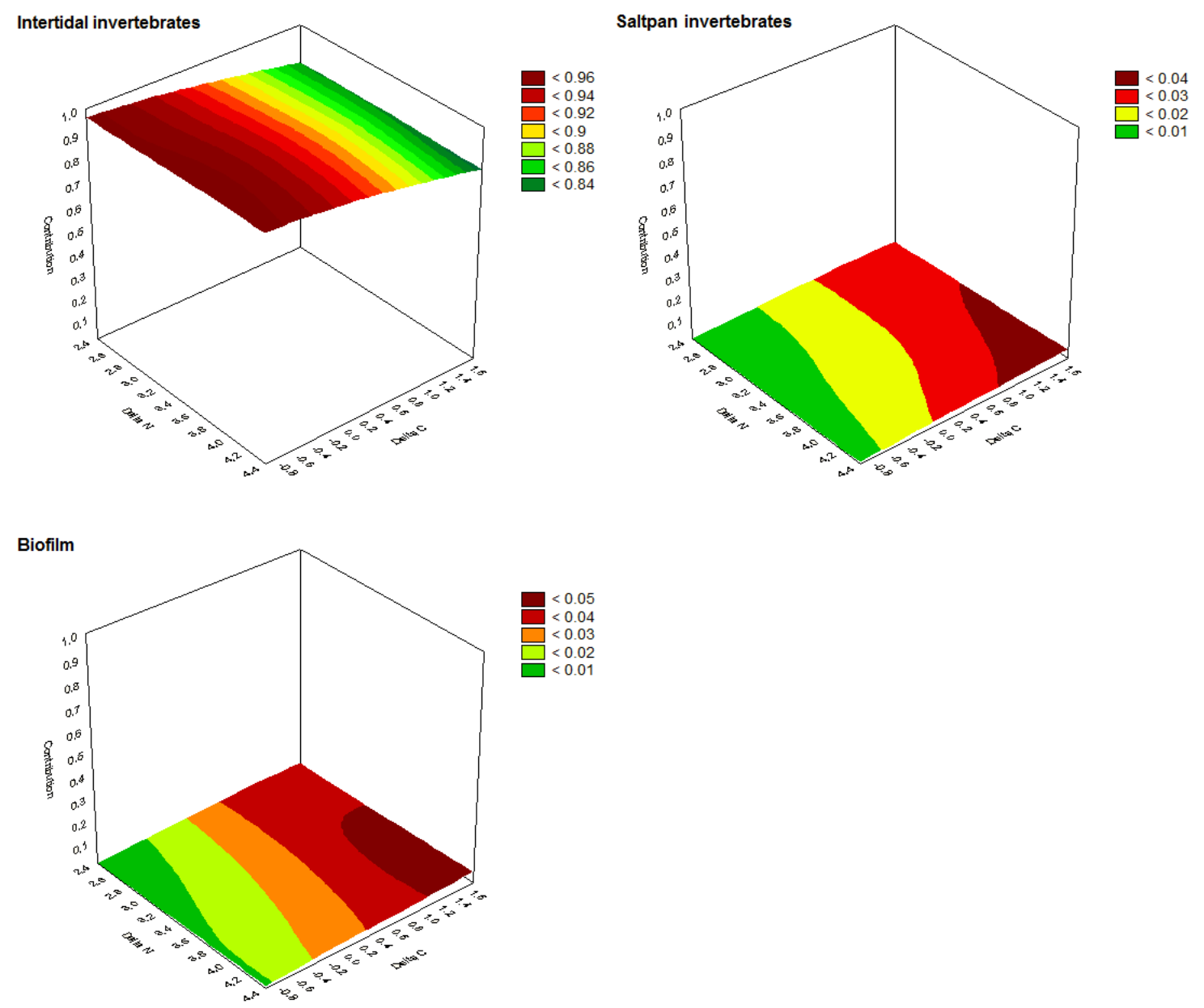


\section{Dunlin (Calidris alpina), Tejo estuary}
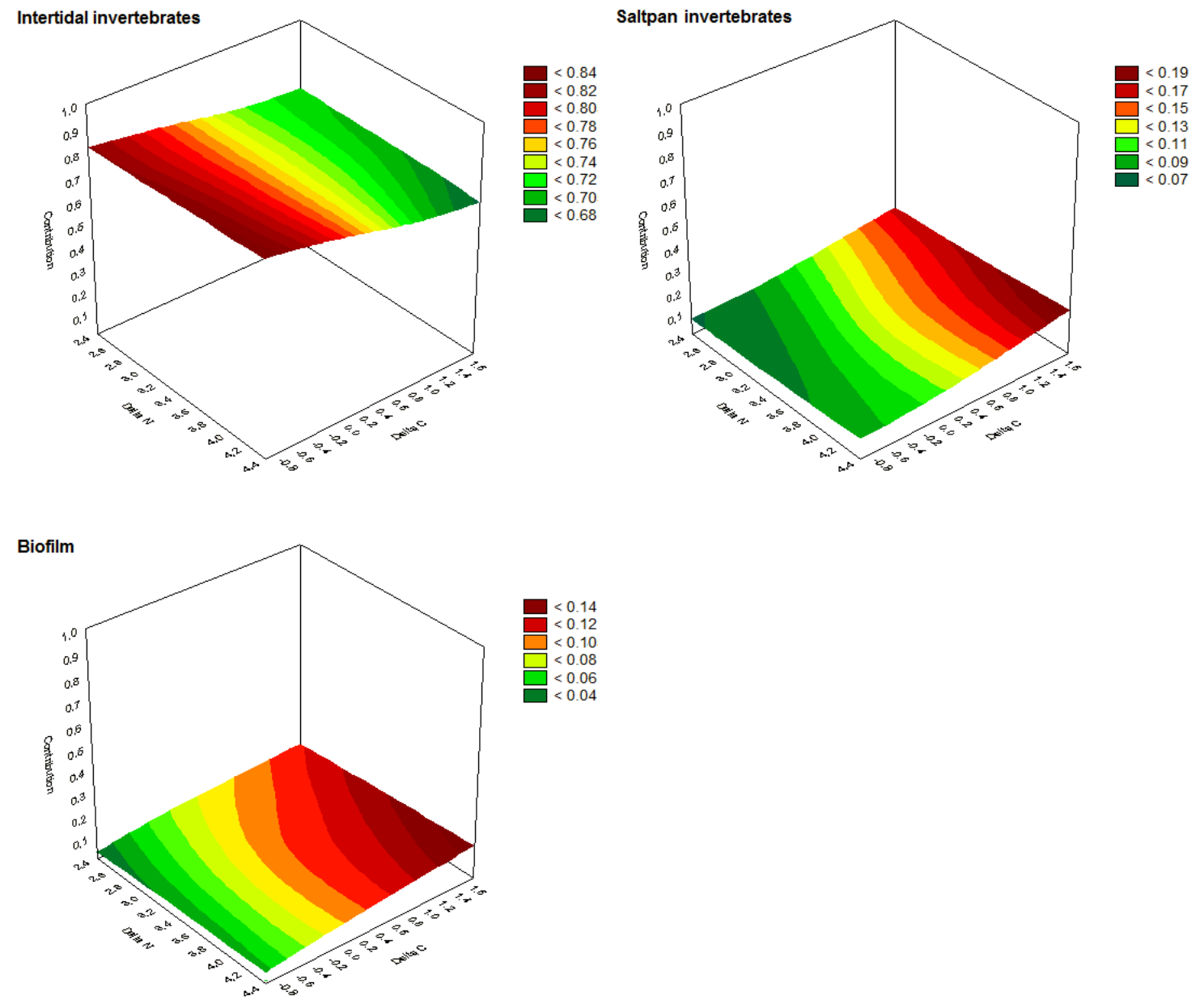
Red knot (Calidris canutus), Tejo estuary
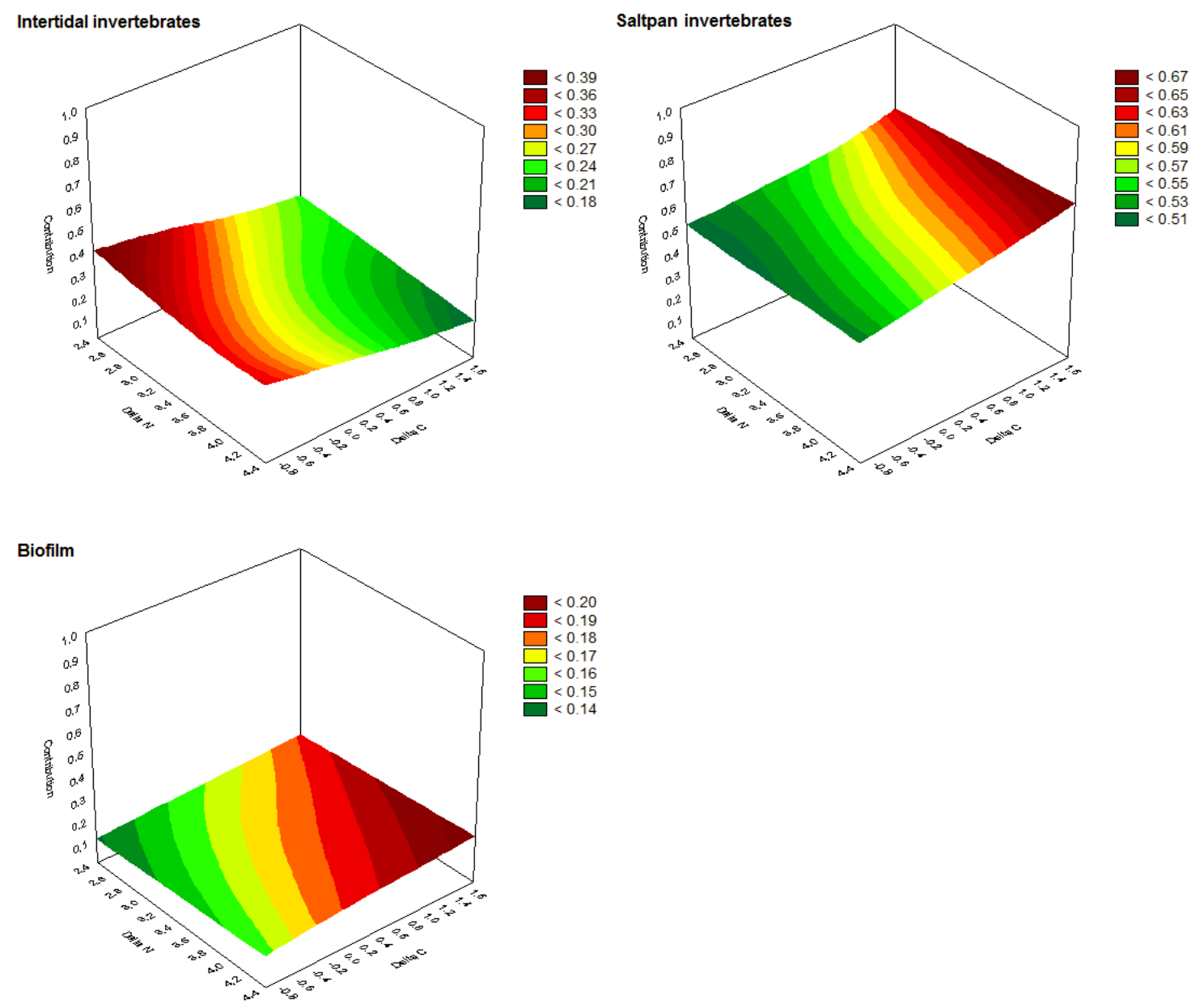


\section{Little stint (Calidris minuta), Tejo estuary}
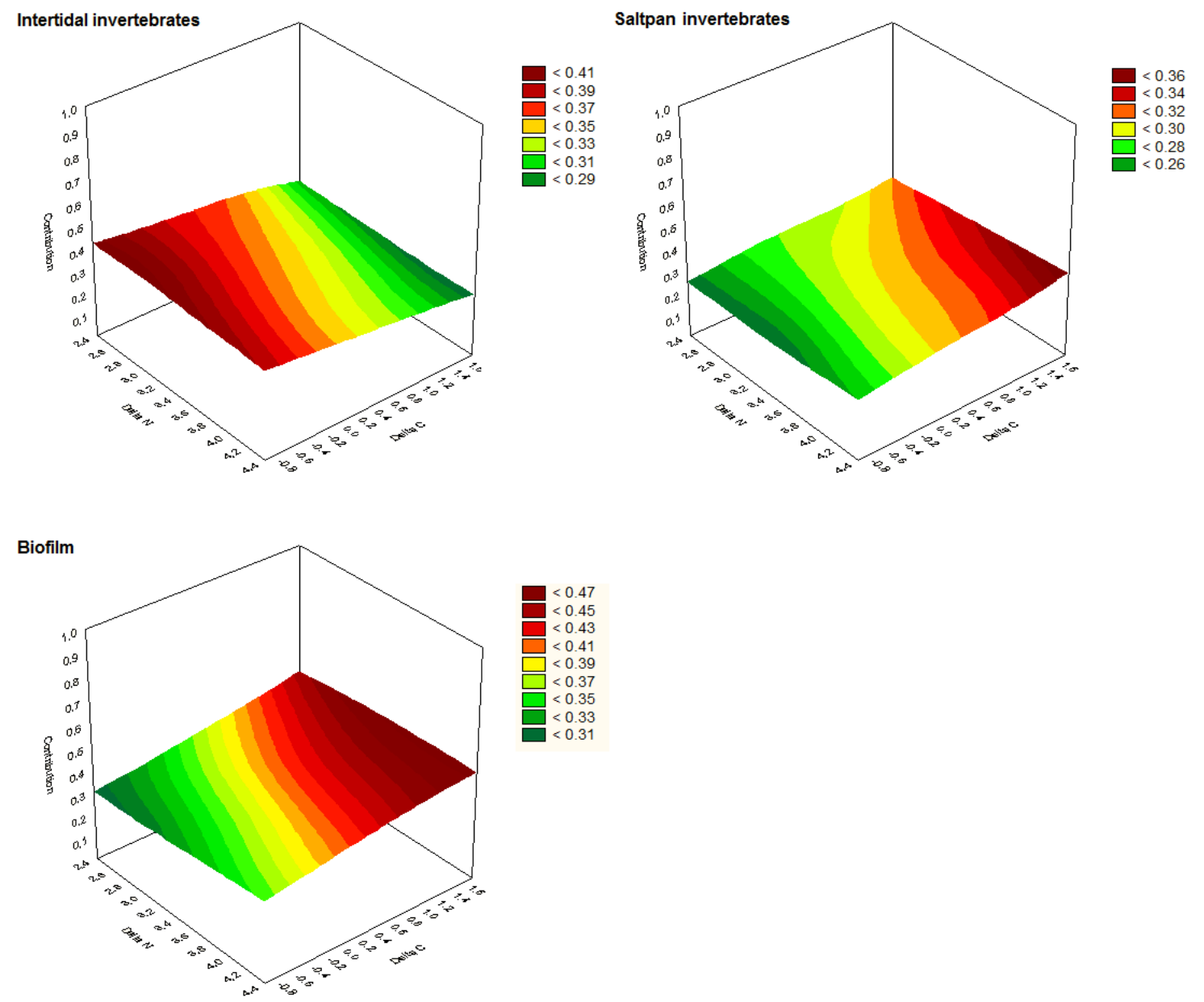
Ringed plover (Charadrius hiaticula), Tejo estuary
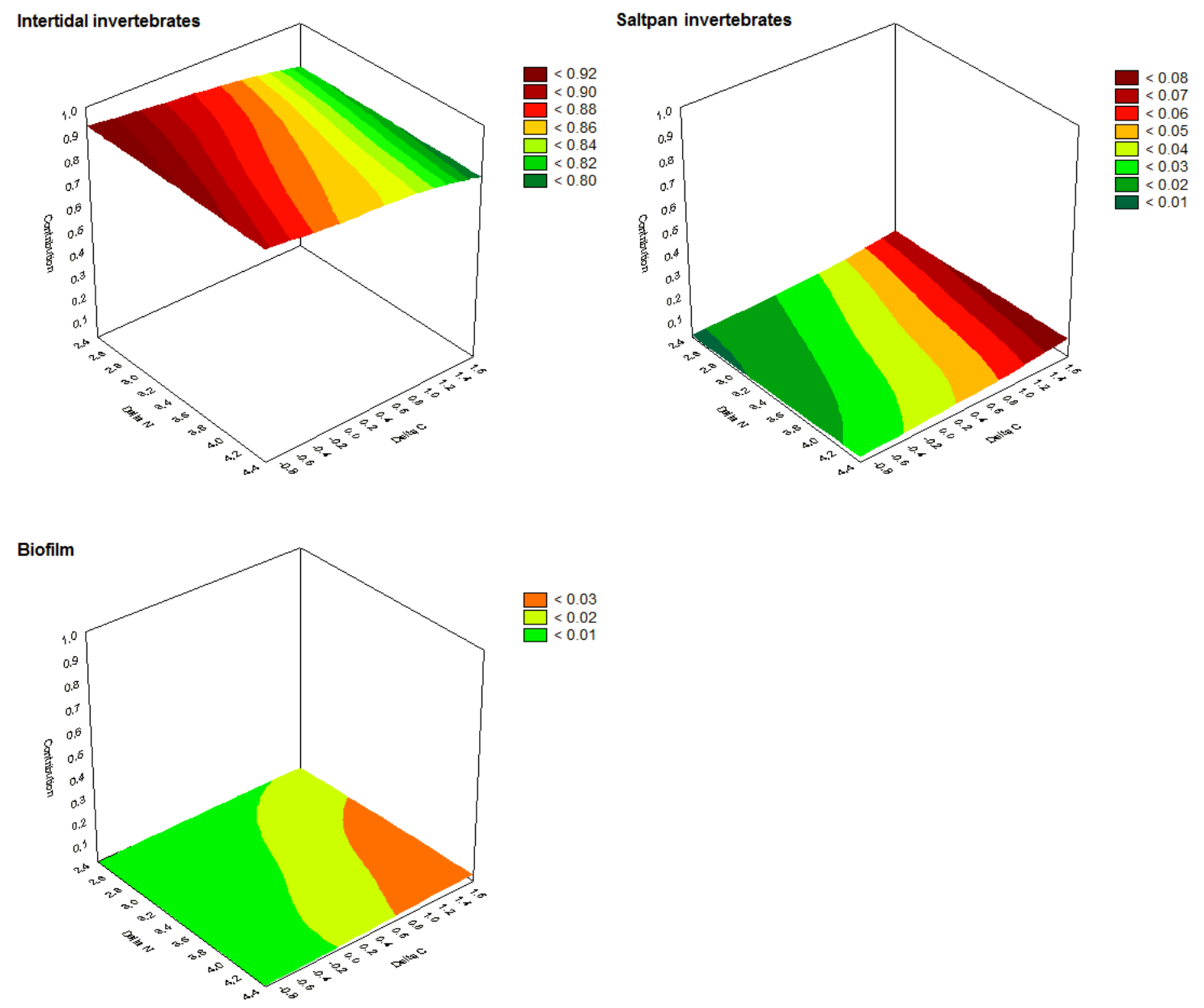
Black-tailed godwit (Limosa limosa), Tejo estuary
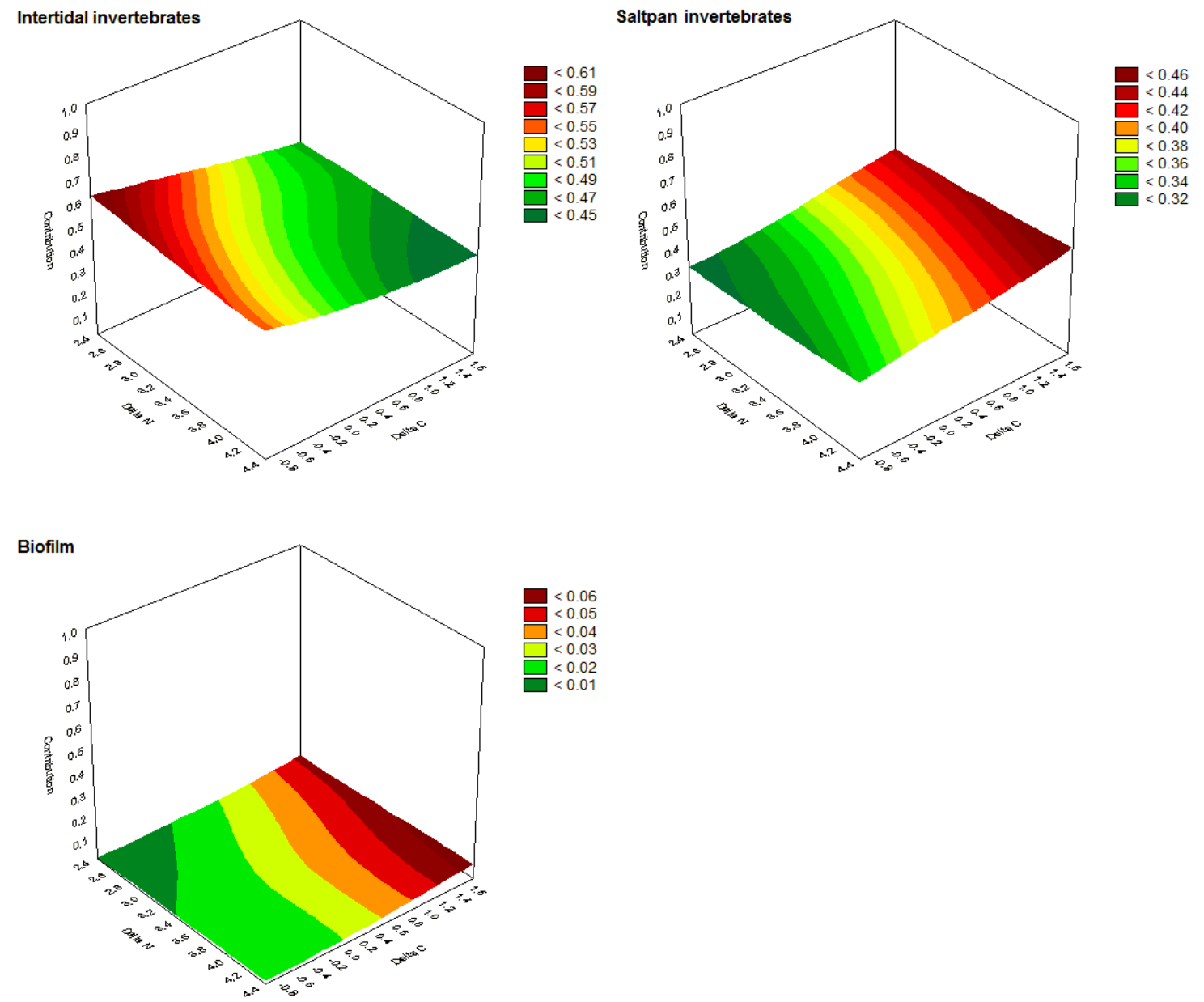
Grey plover (Pluvialis squatarola), Tejo estuary
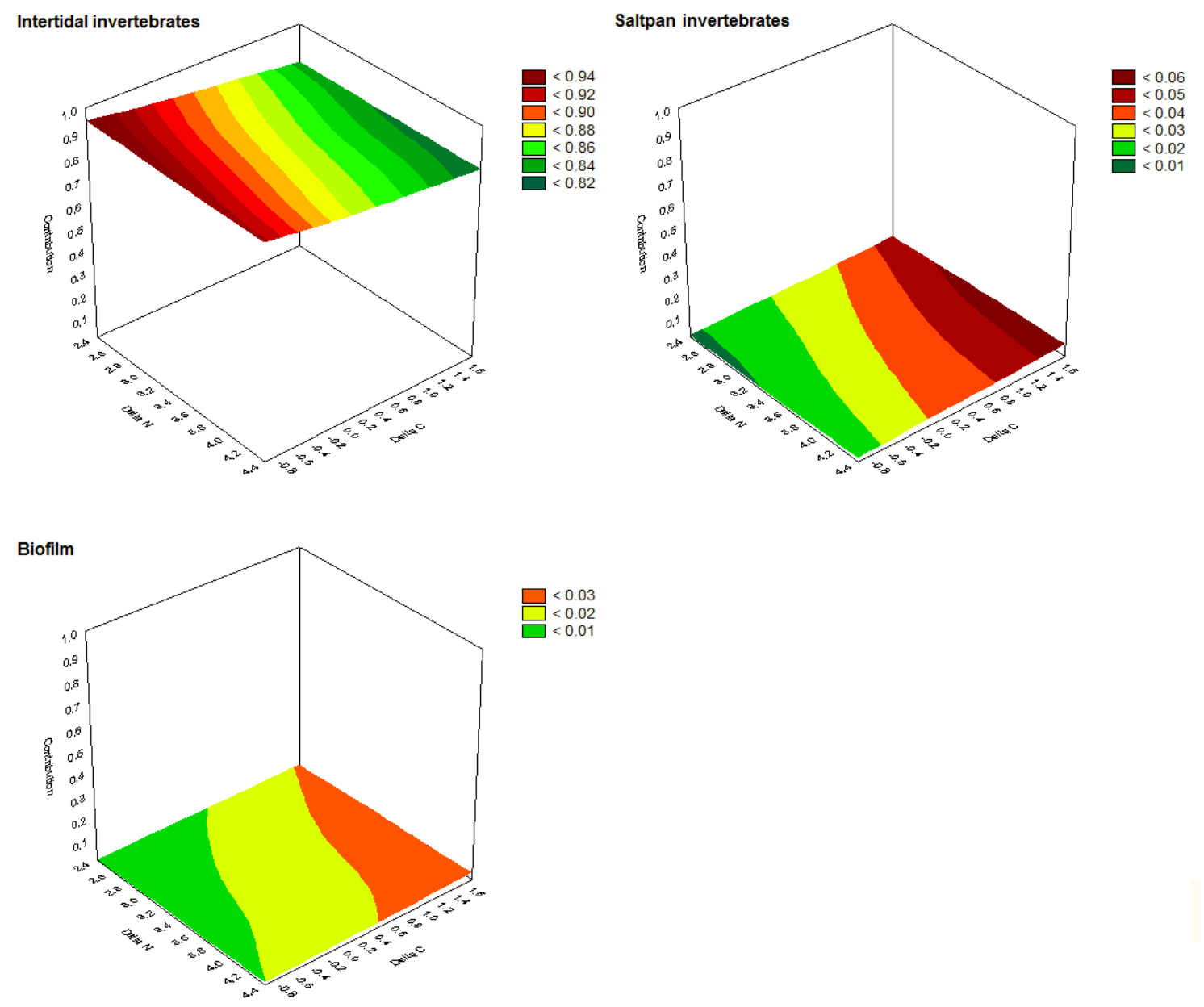

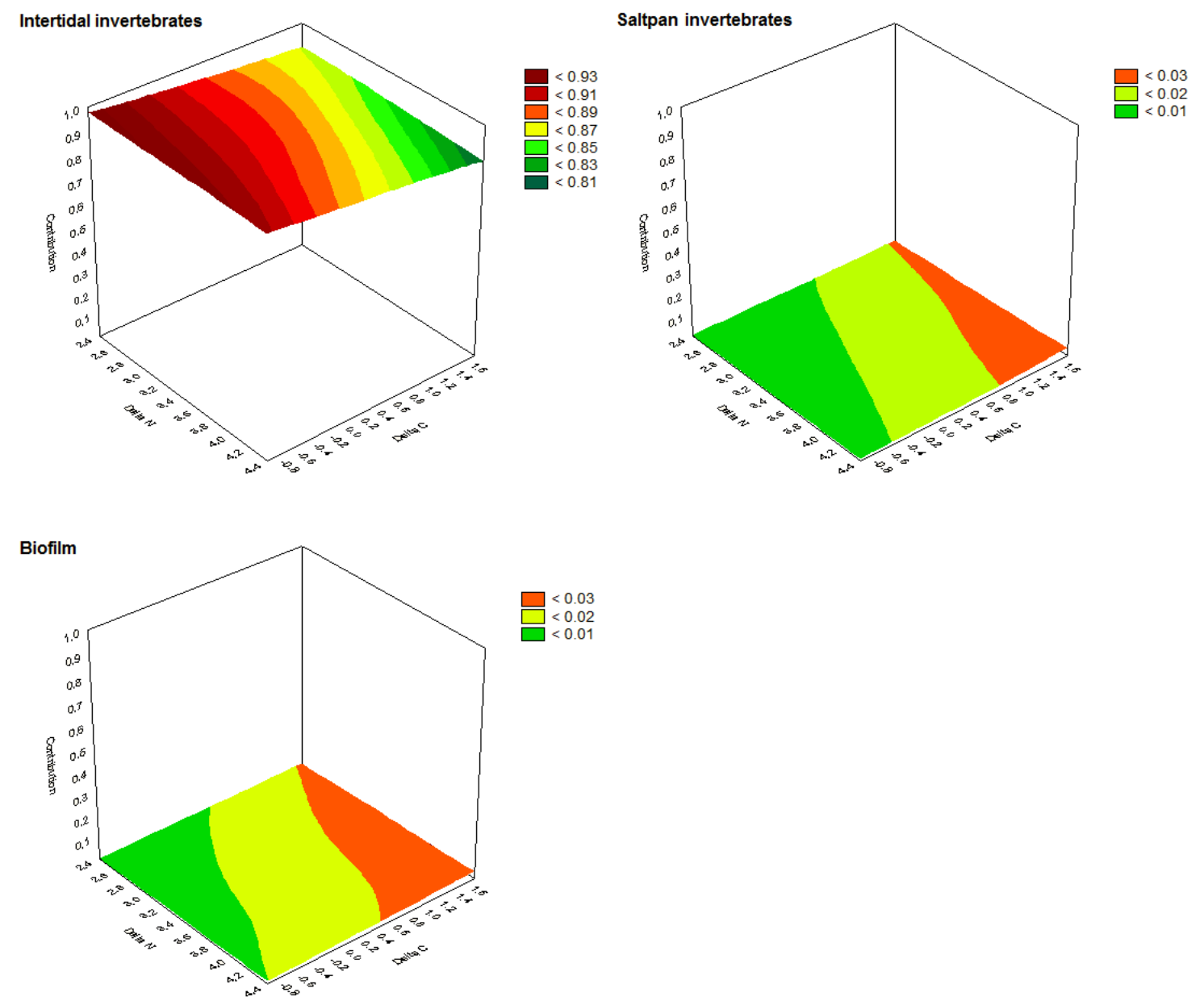

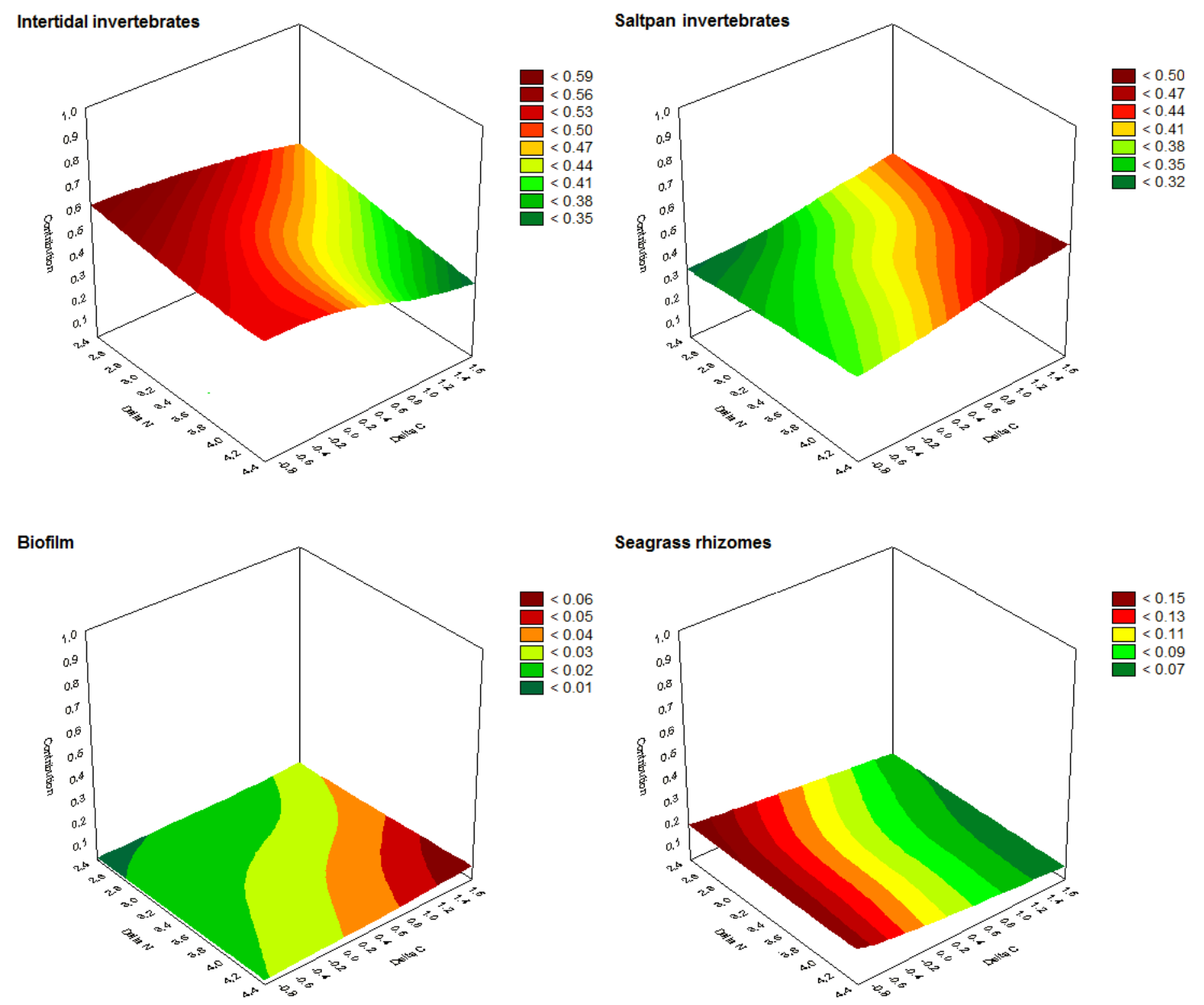

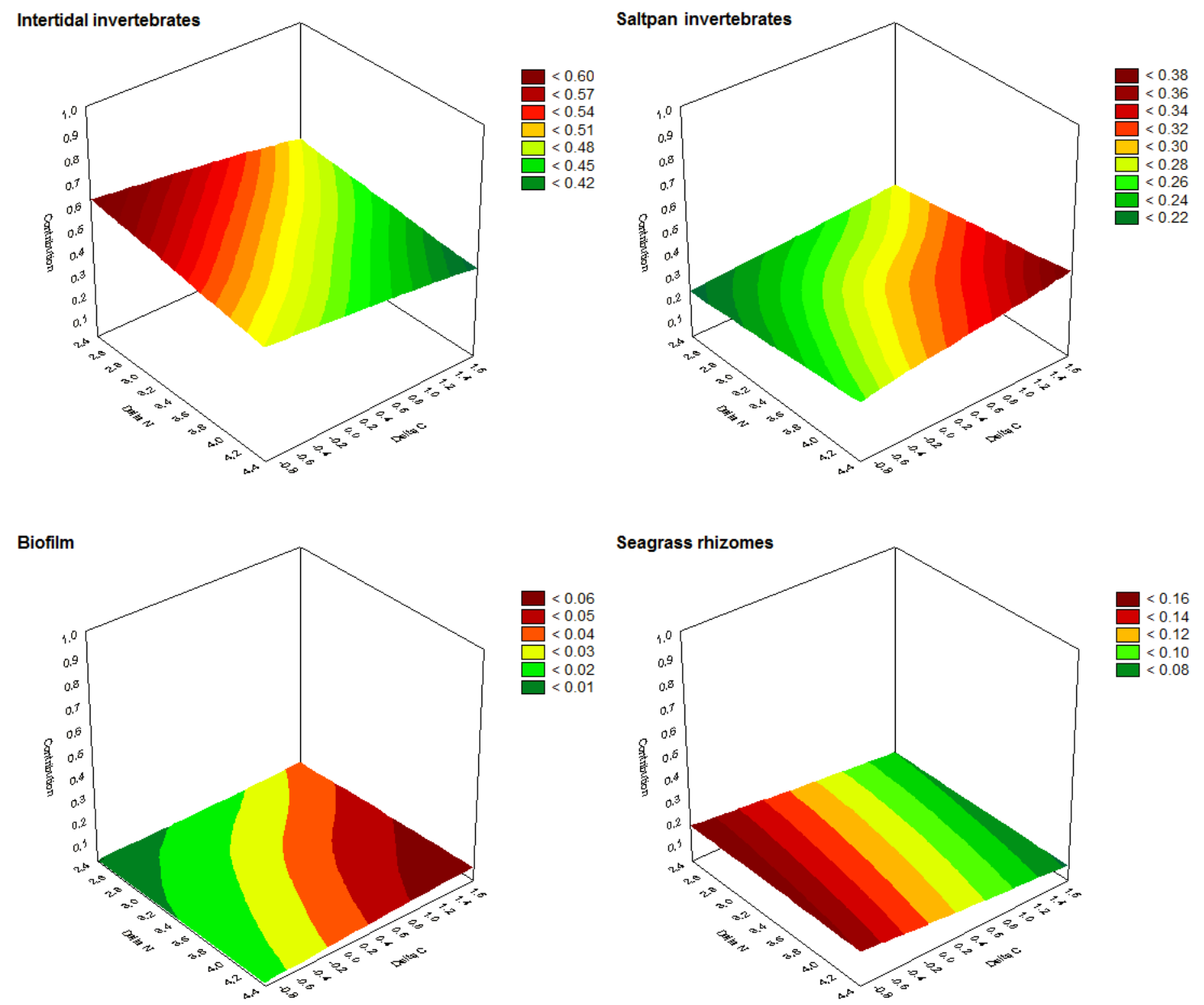


\section{Little stint (Calidris minuta), Sidi-Moussa}
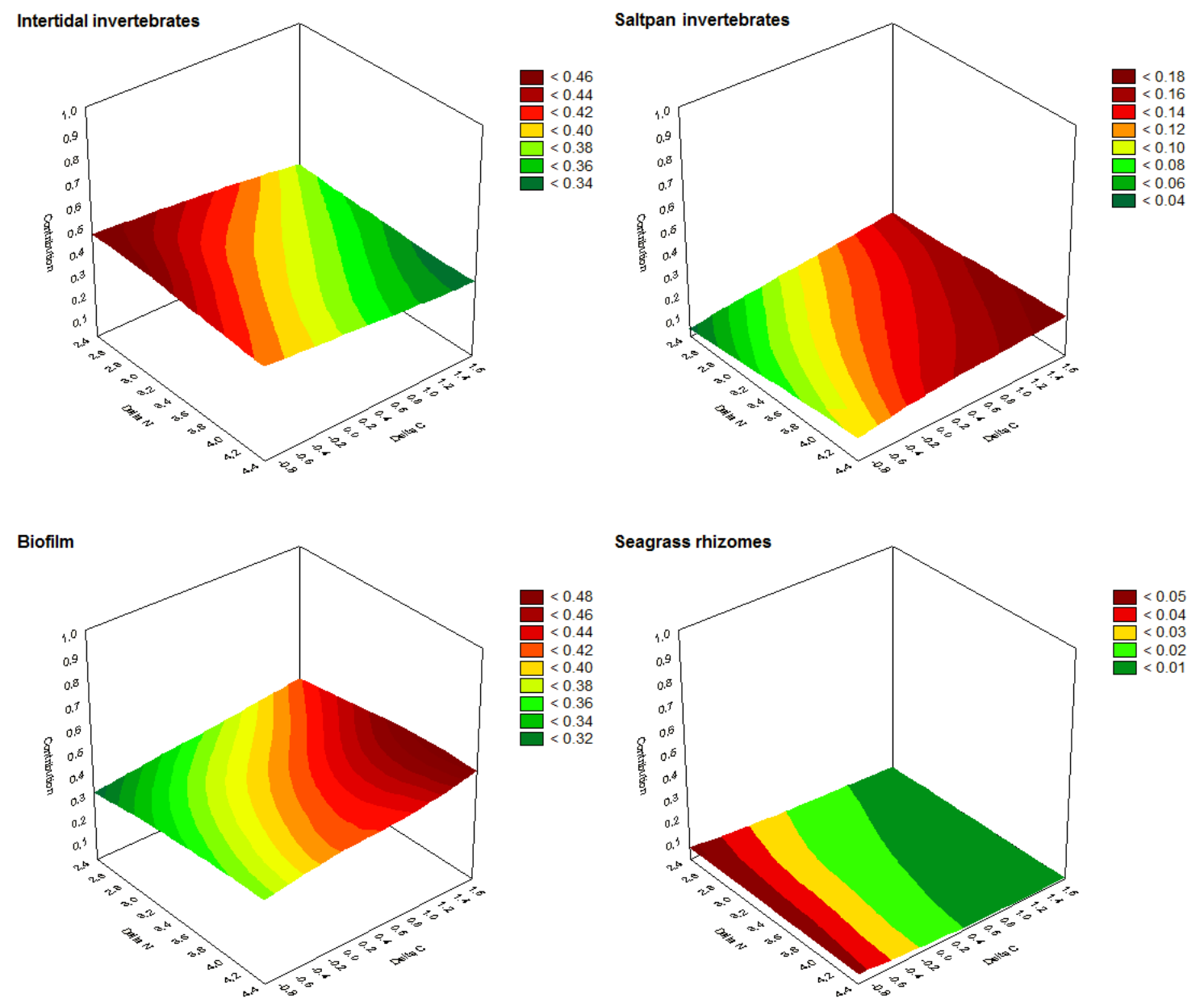
Ringed plover (Charadrius hiaticula), Sidi-Moussa
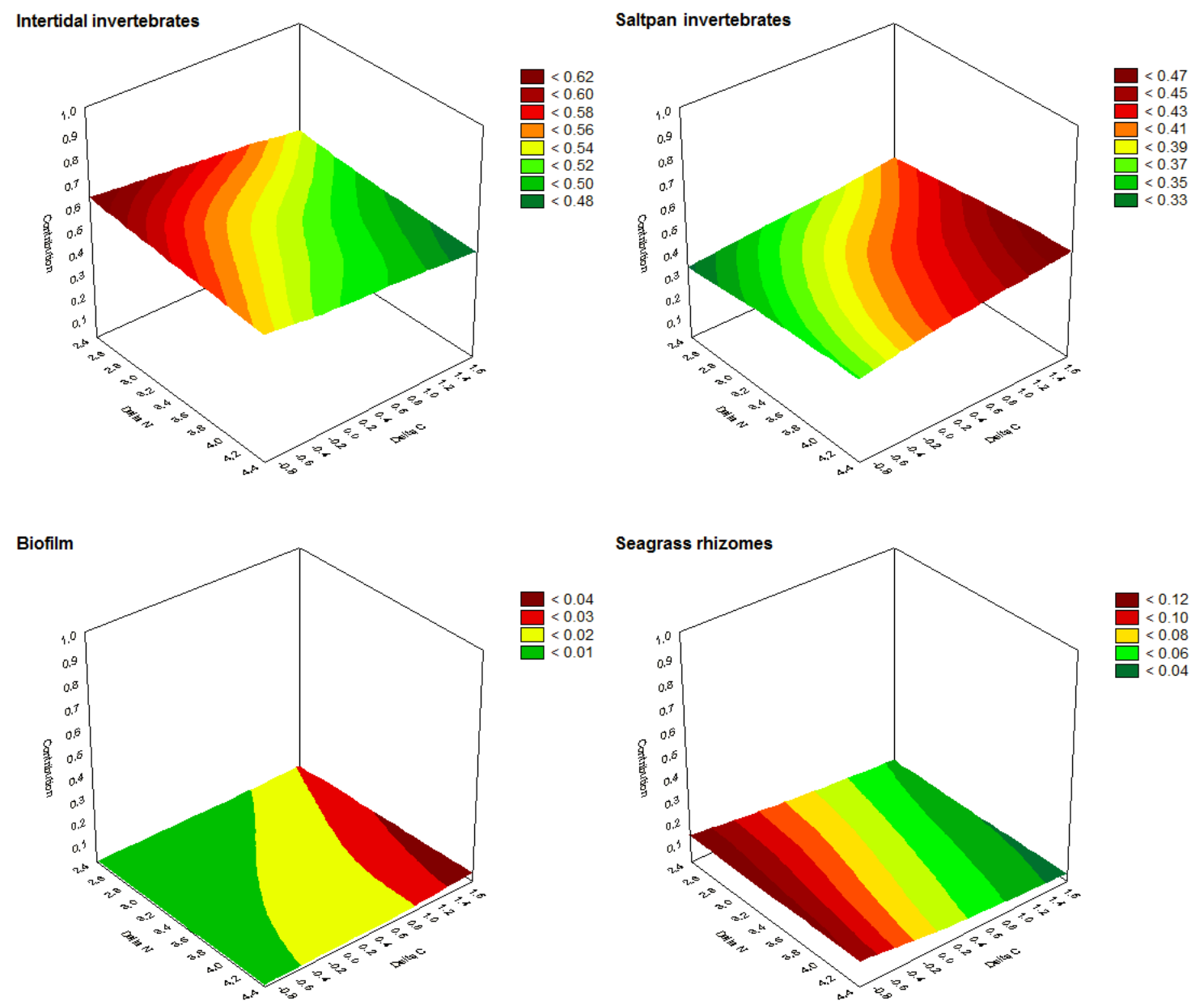

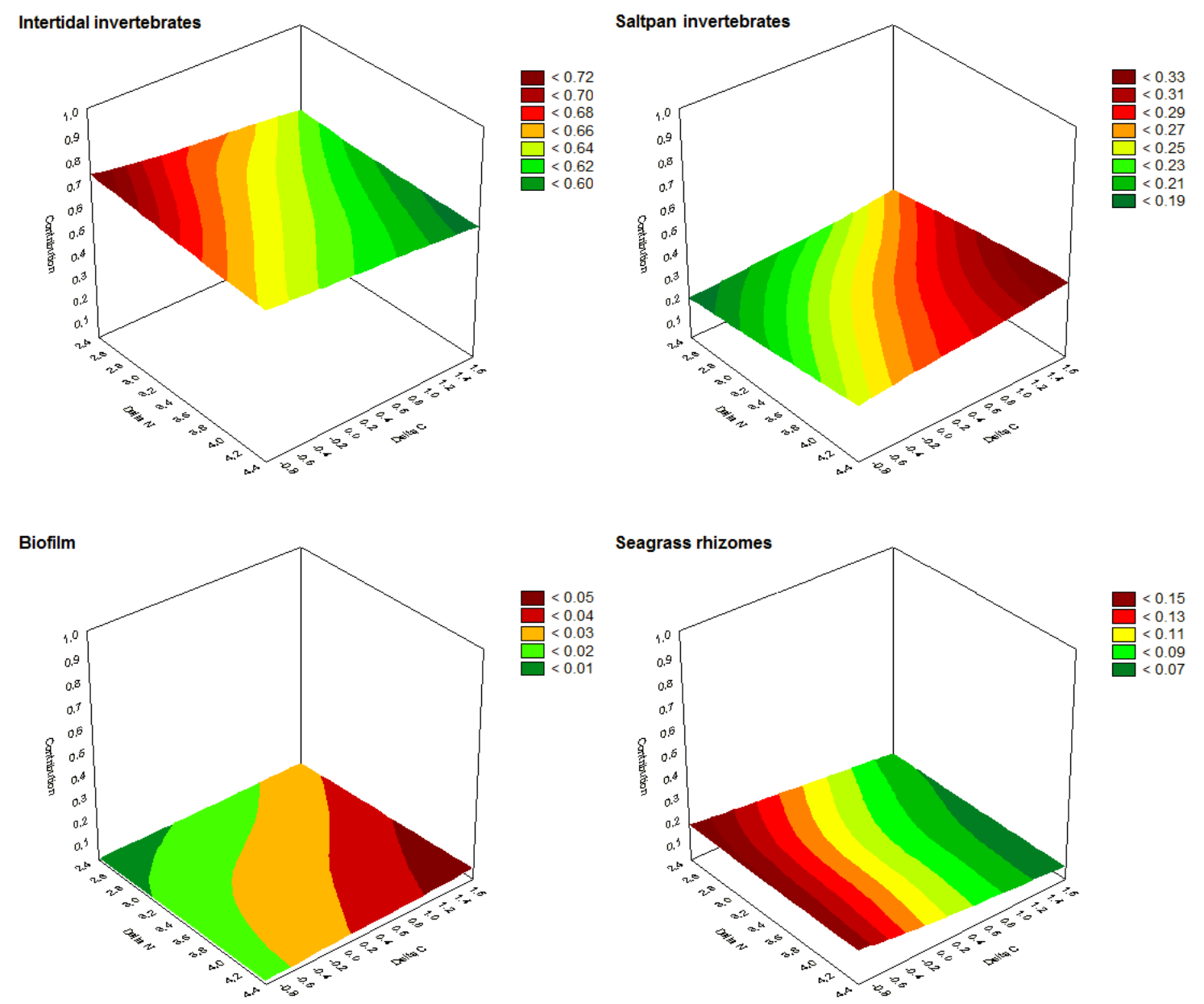

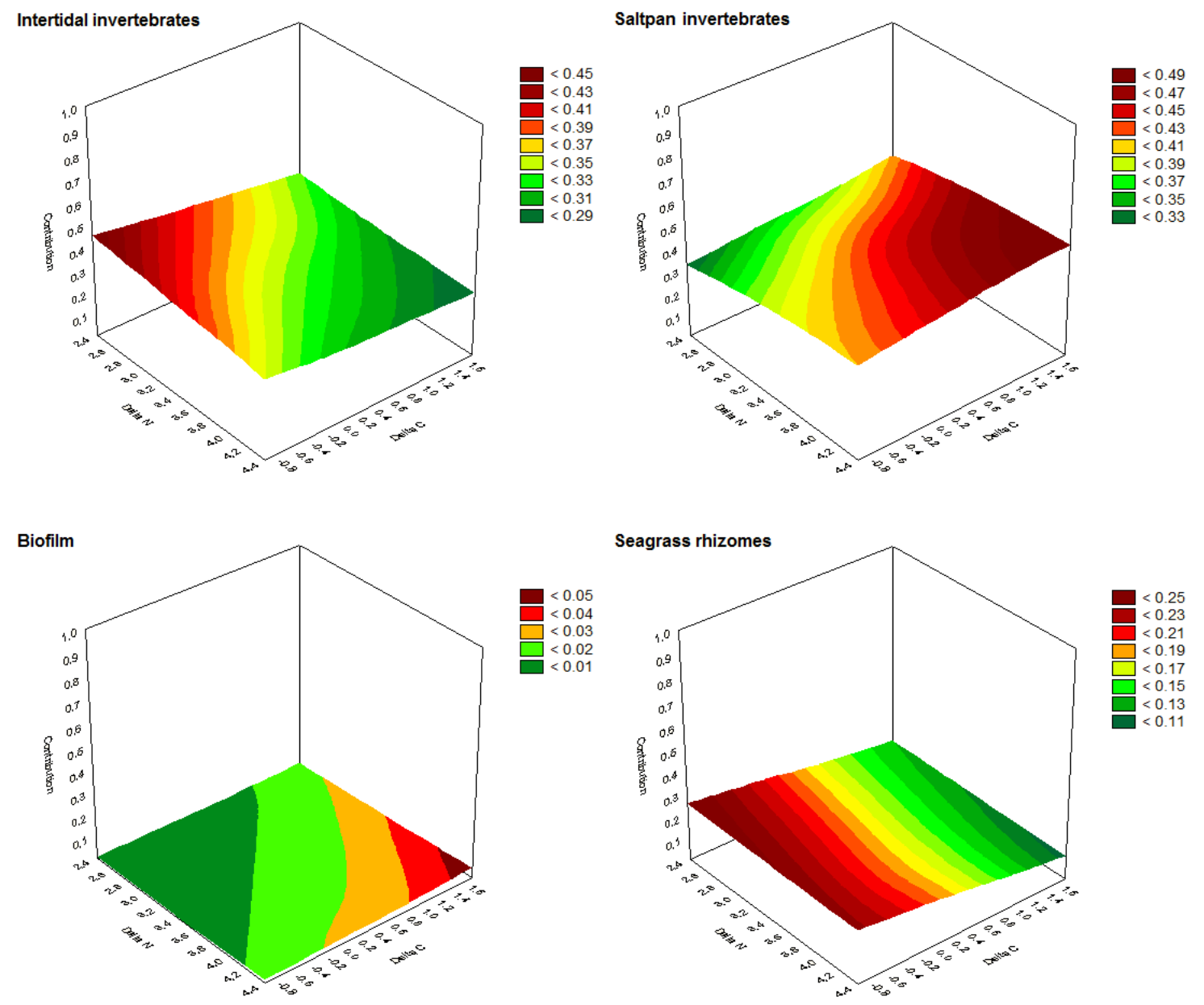
Ruddy turnstone (Arenaria interpres), Banc d'Arguin
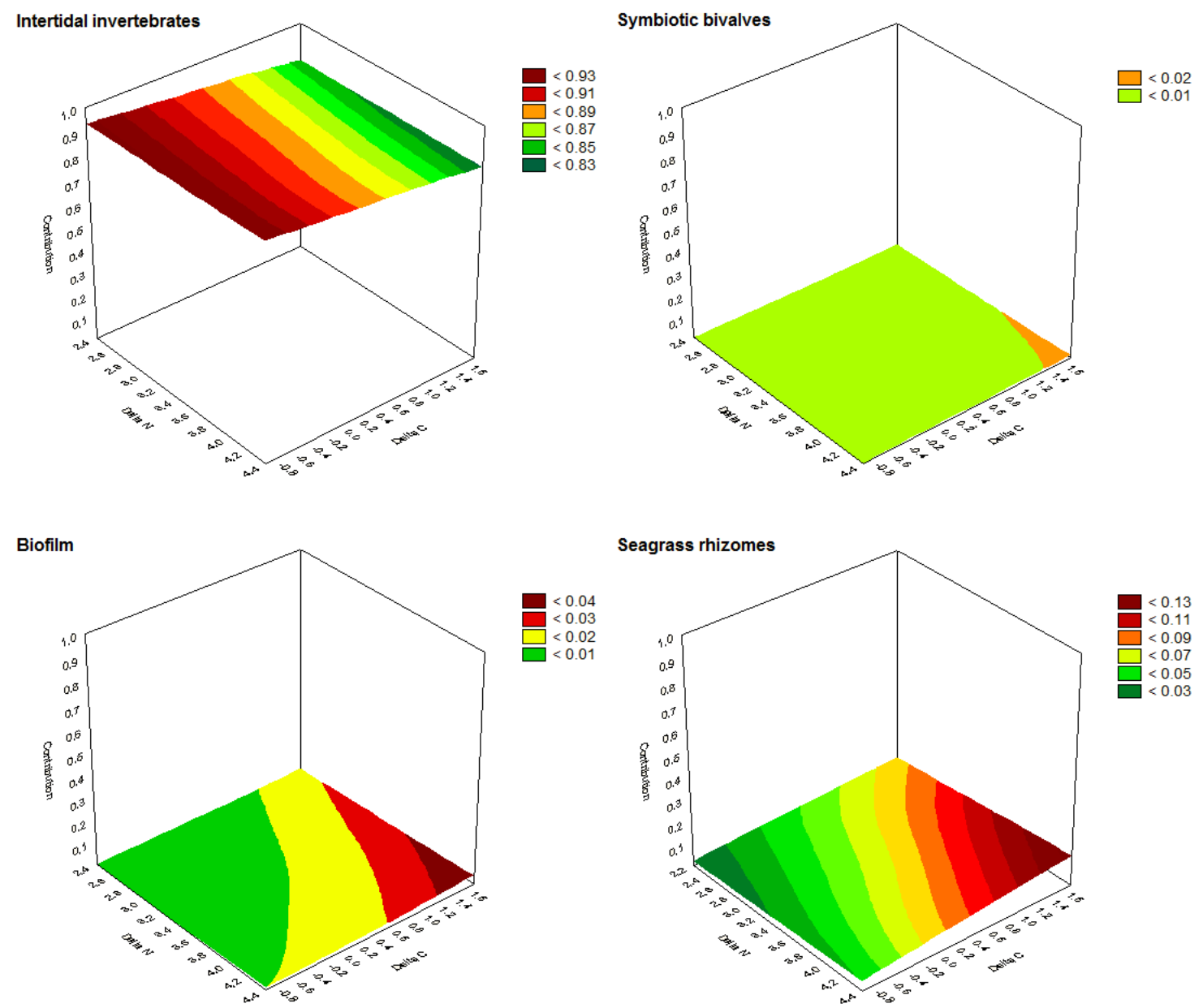

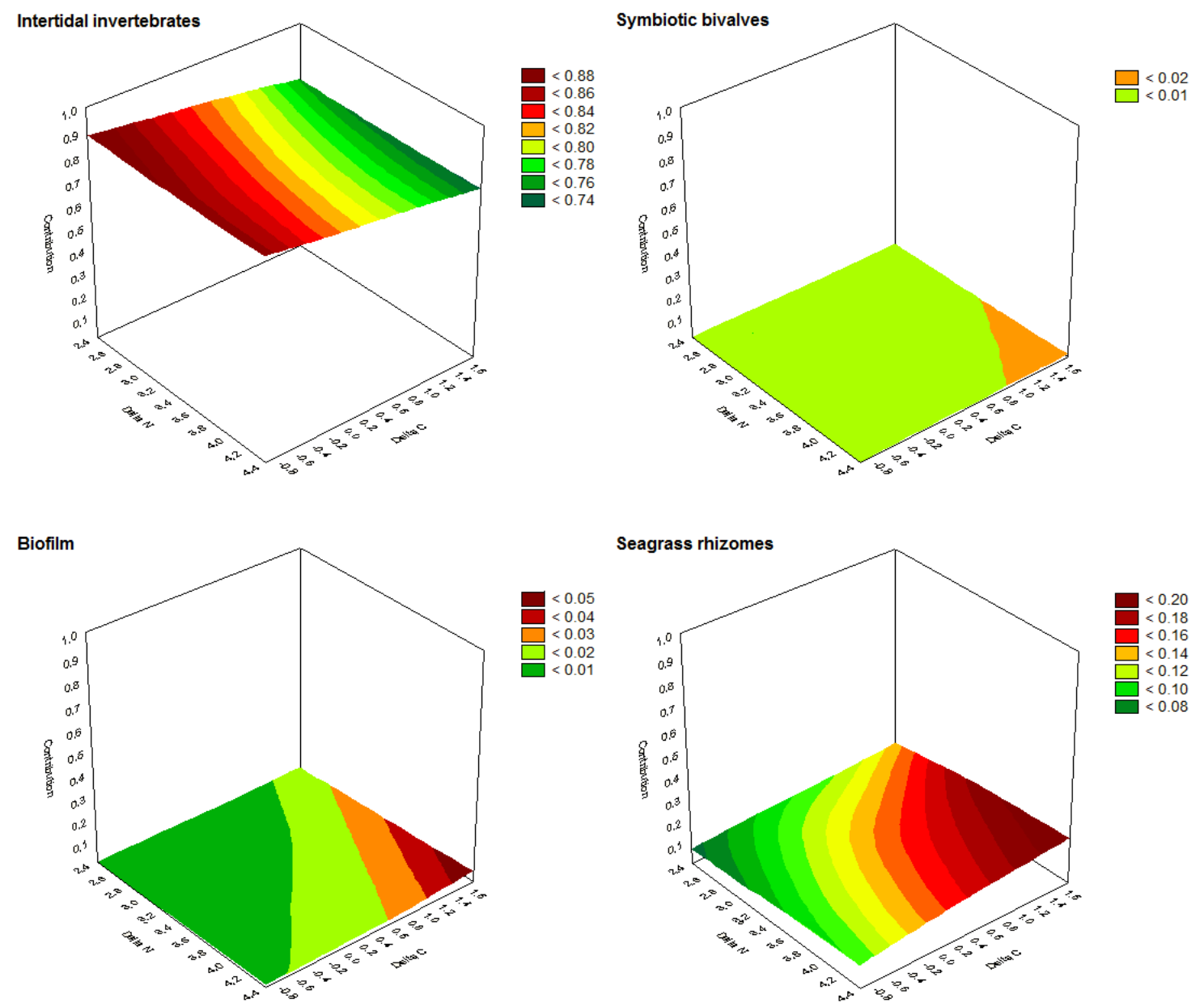

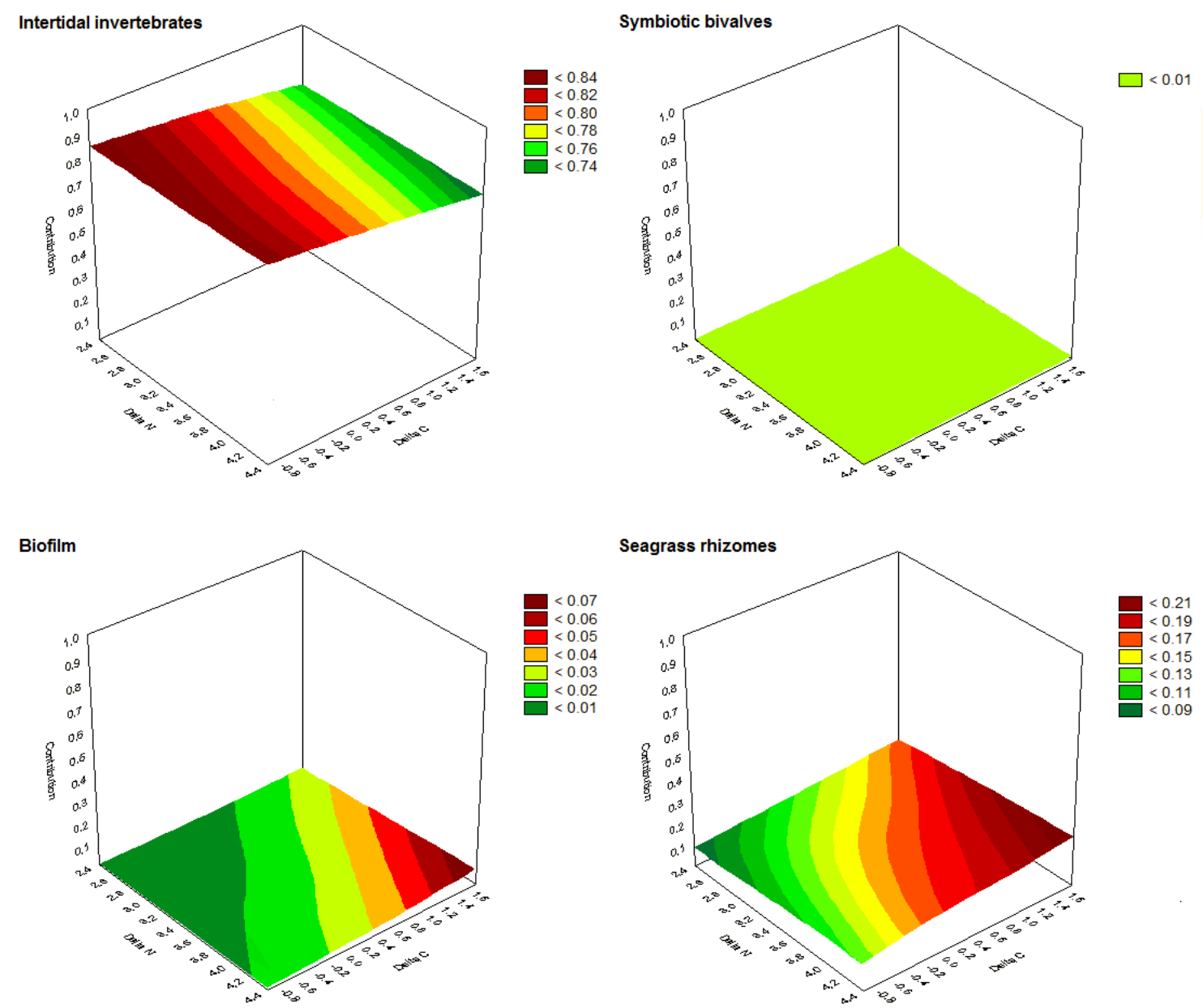
Red knot (Calidris canutus), Banc d'Arguin
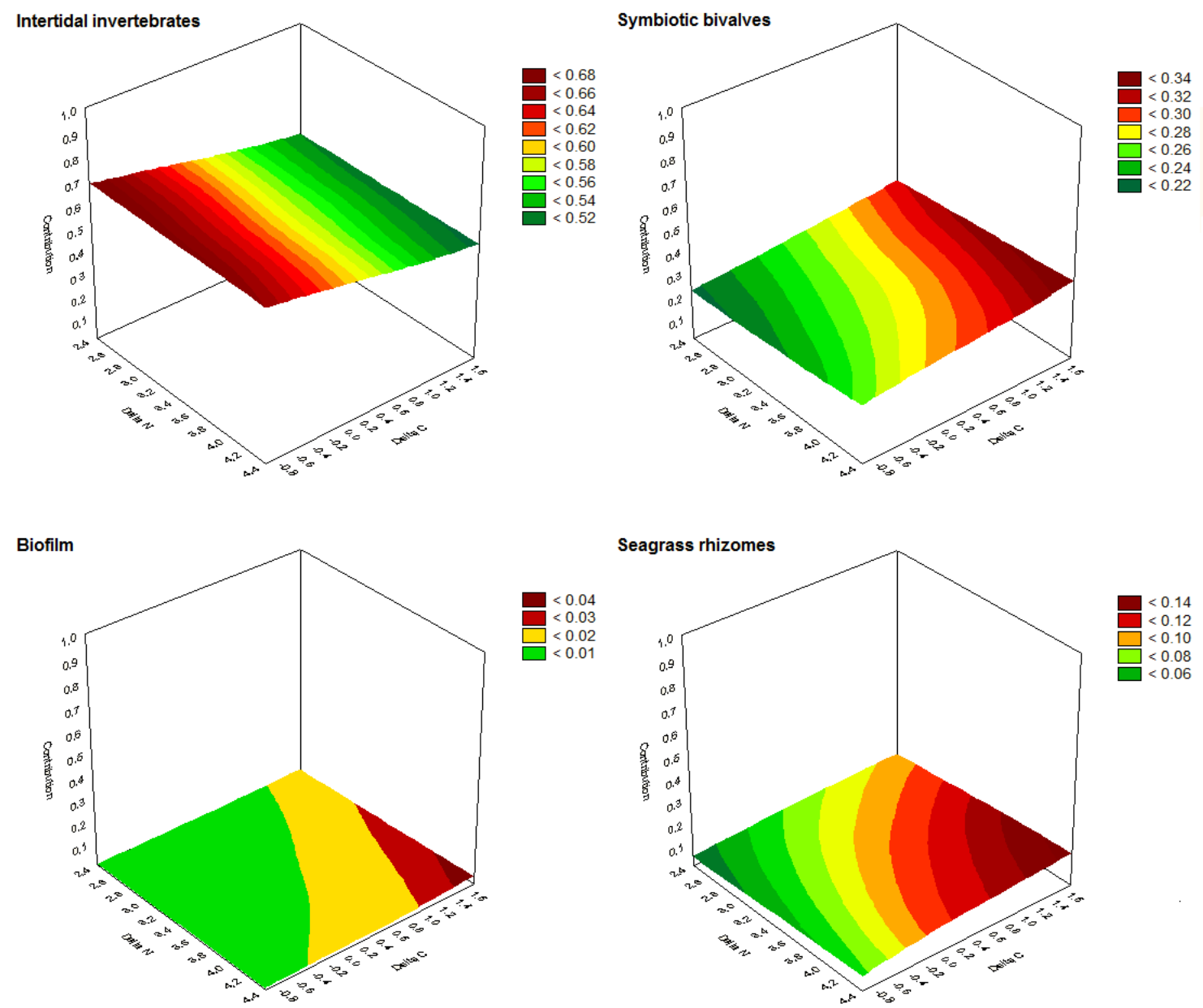

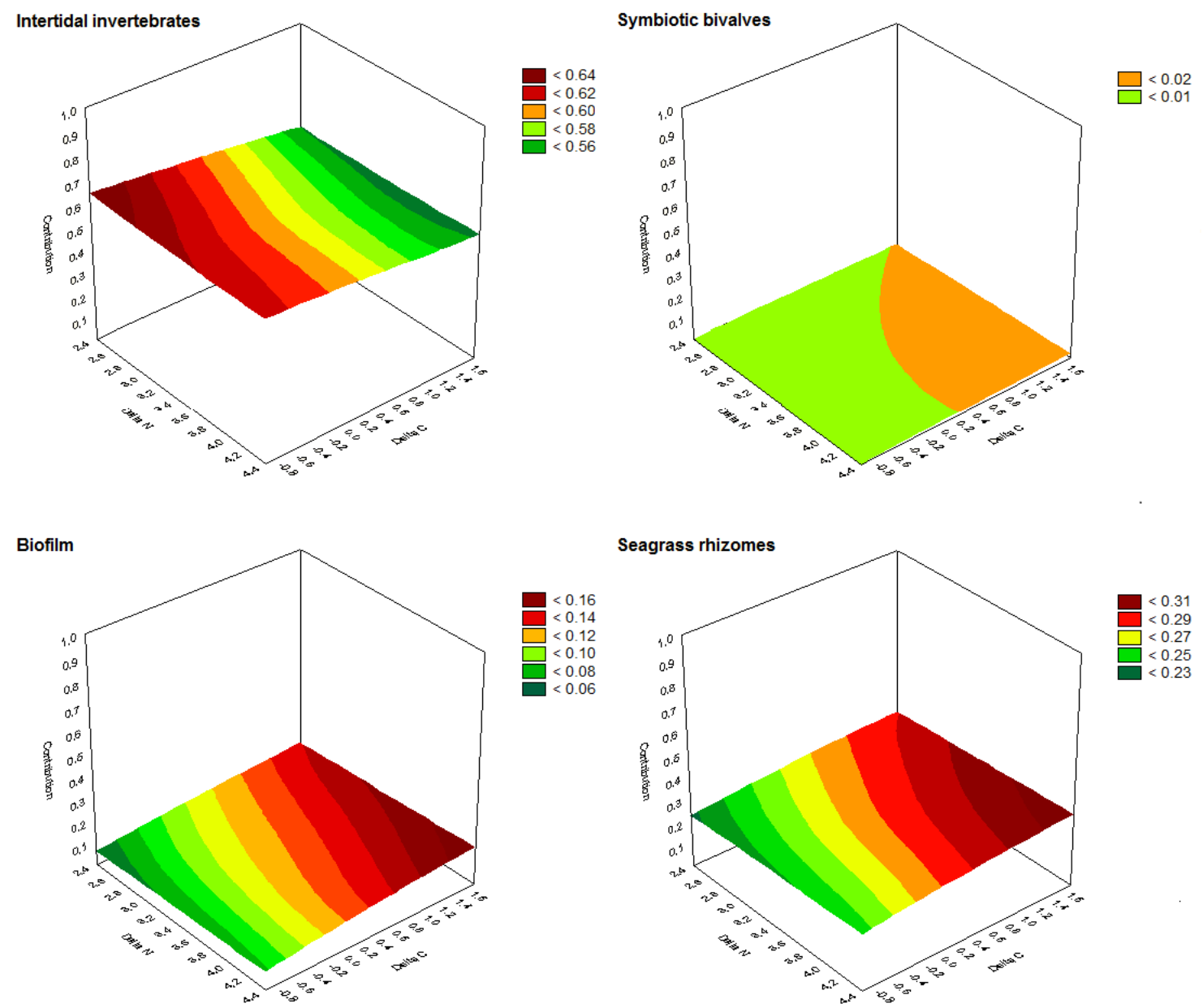
Intertidal invertebrates

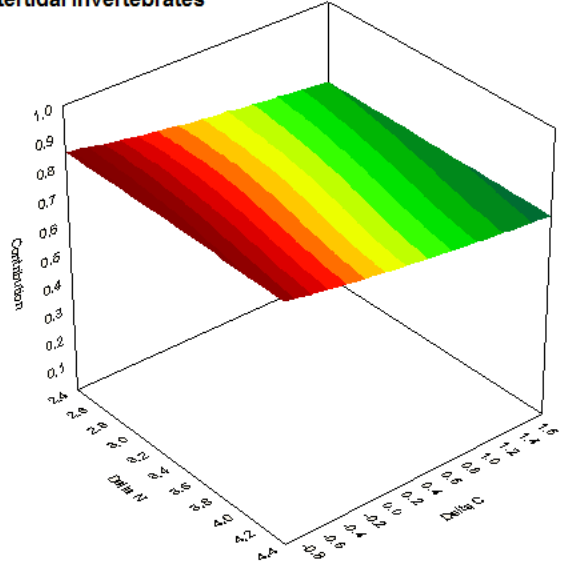

Biofilm

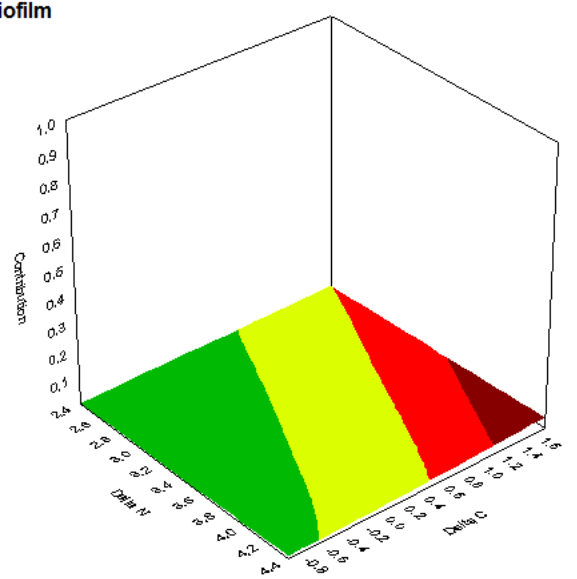

Symbiotic bivalves
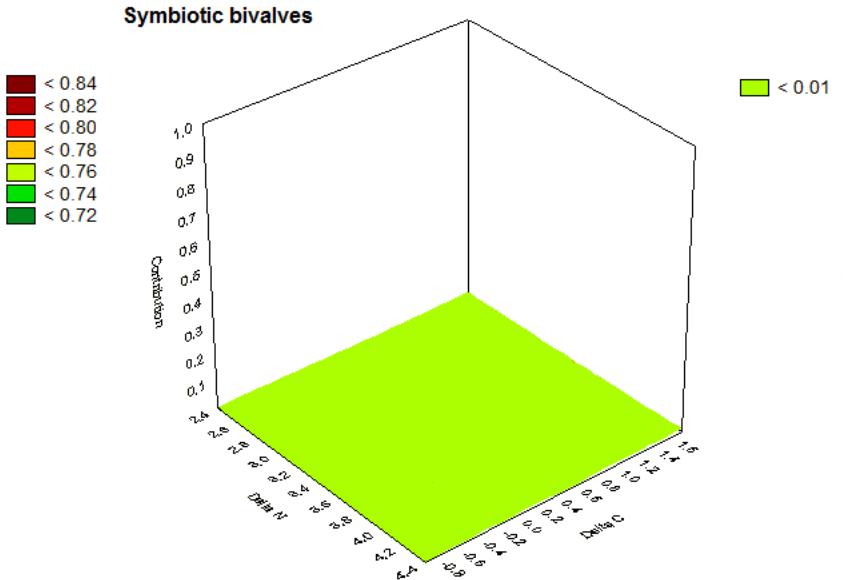

Seagrass rhizomes
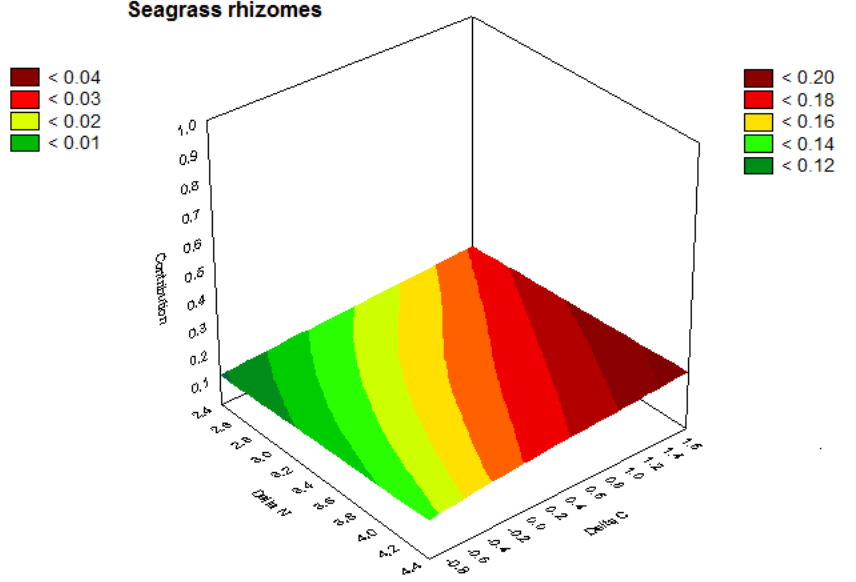


\section{Ringed plover (Charadrius hiaticula), Banc d'Arguin}
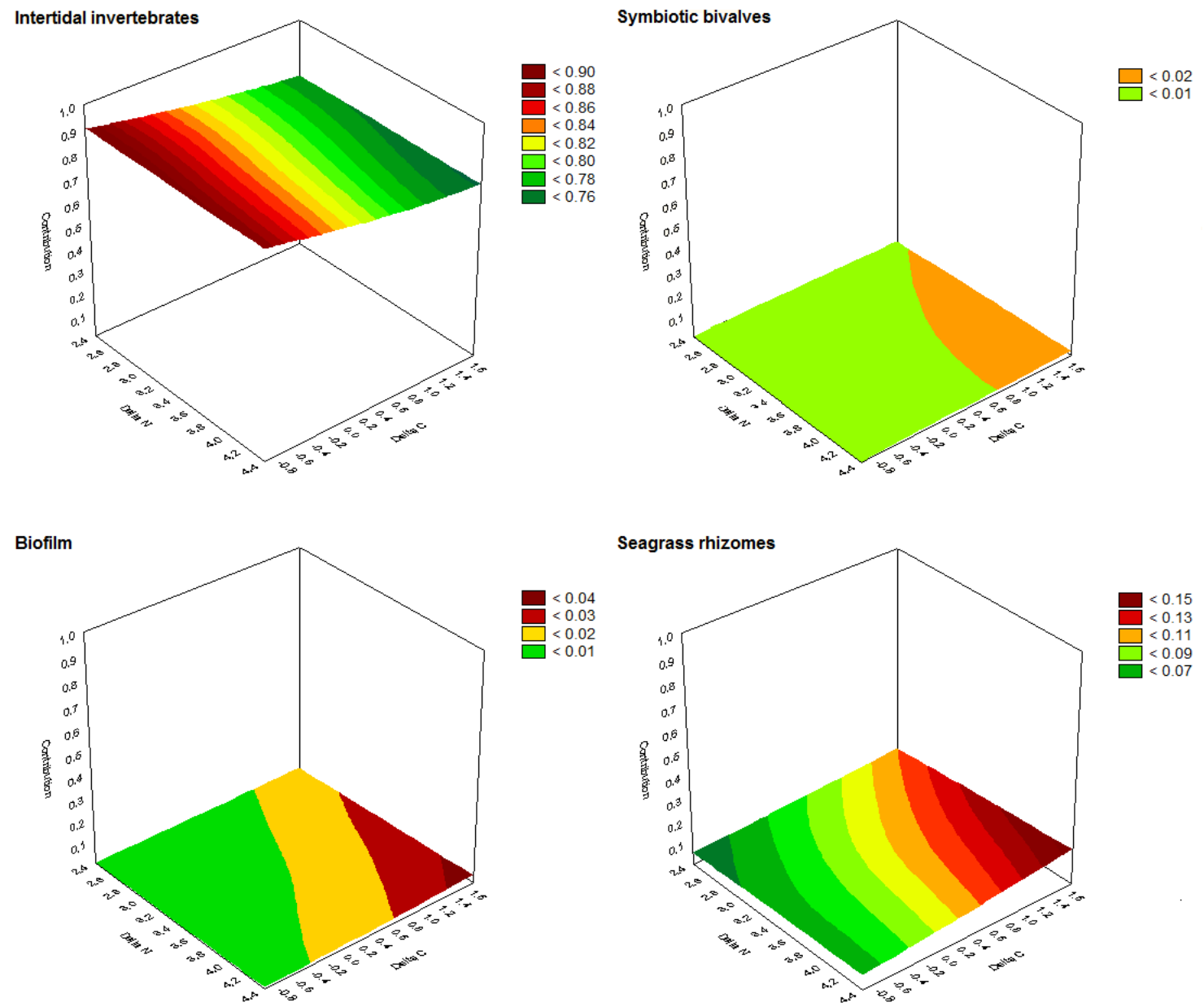

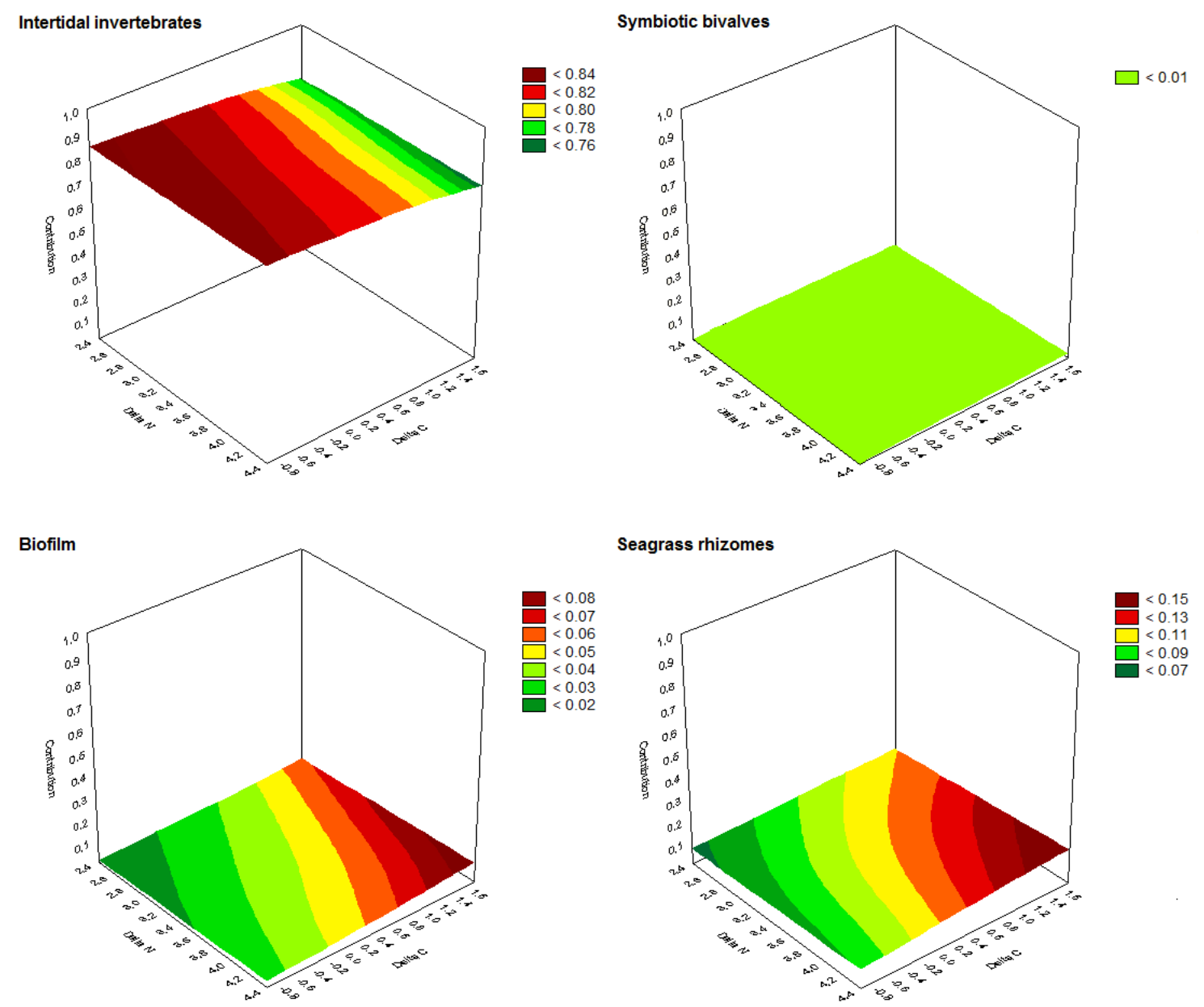

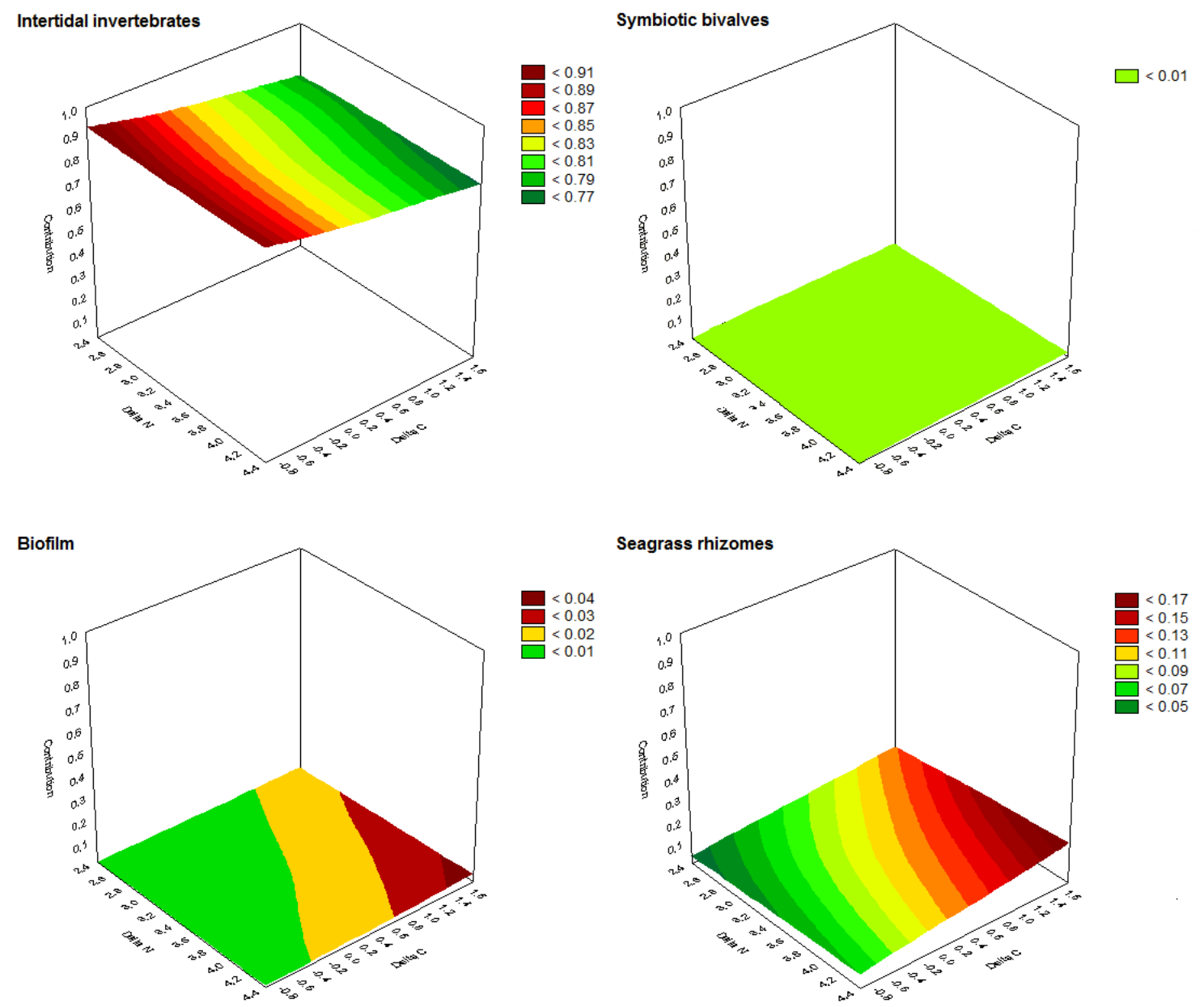
Ruddy turnstone (Arenaria interpres), Bijagós archipelago
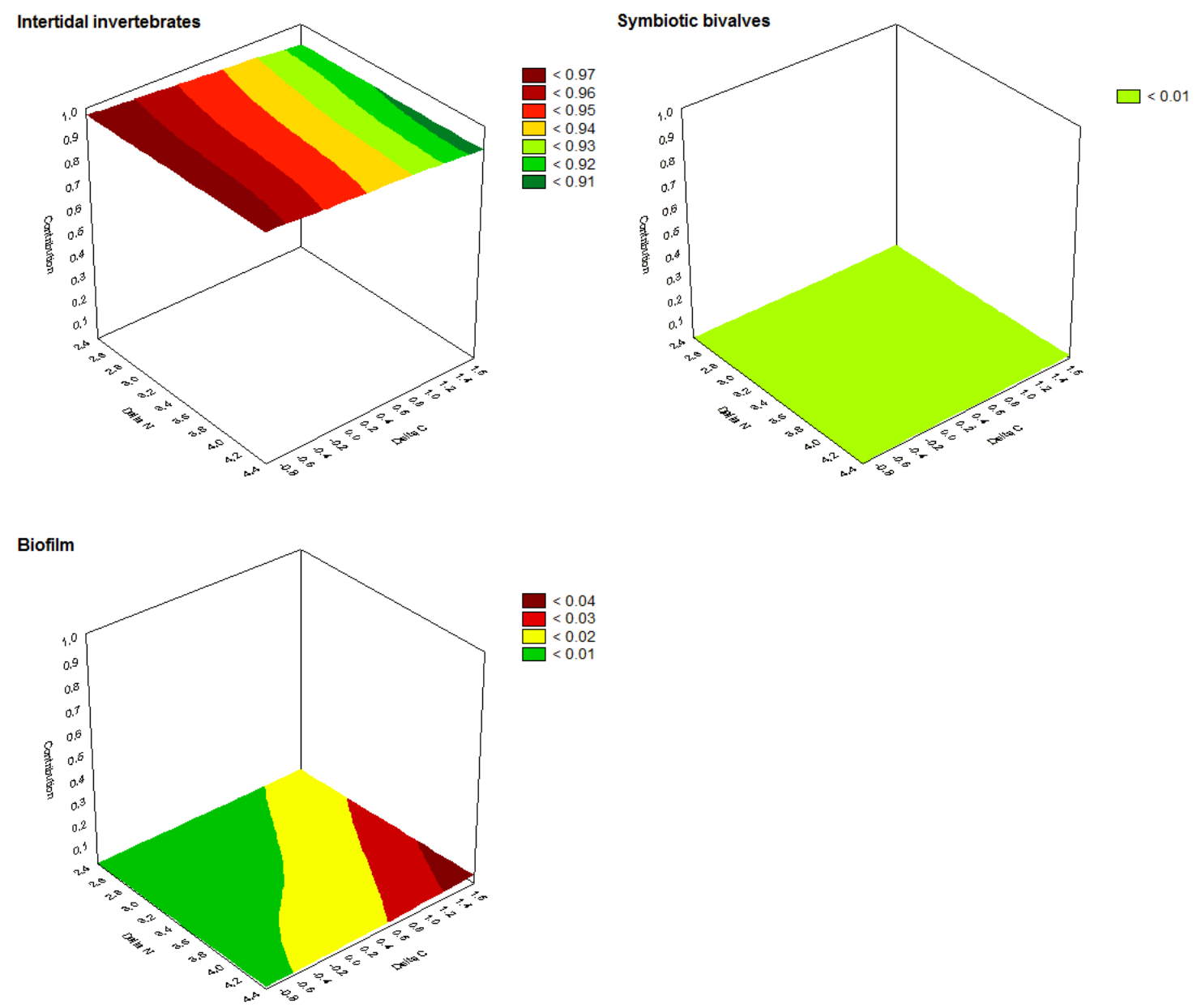
Sanderling (Calidris alba), Bijagós archipelago
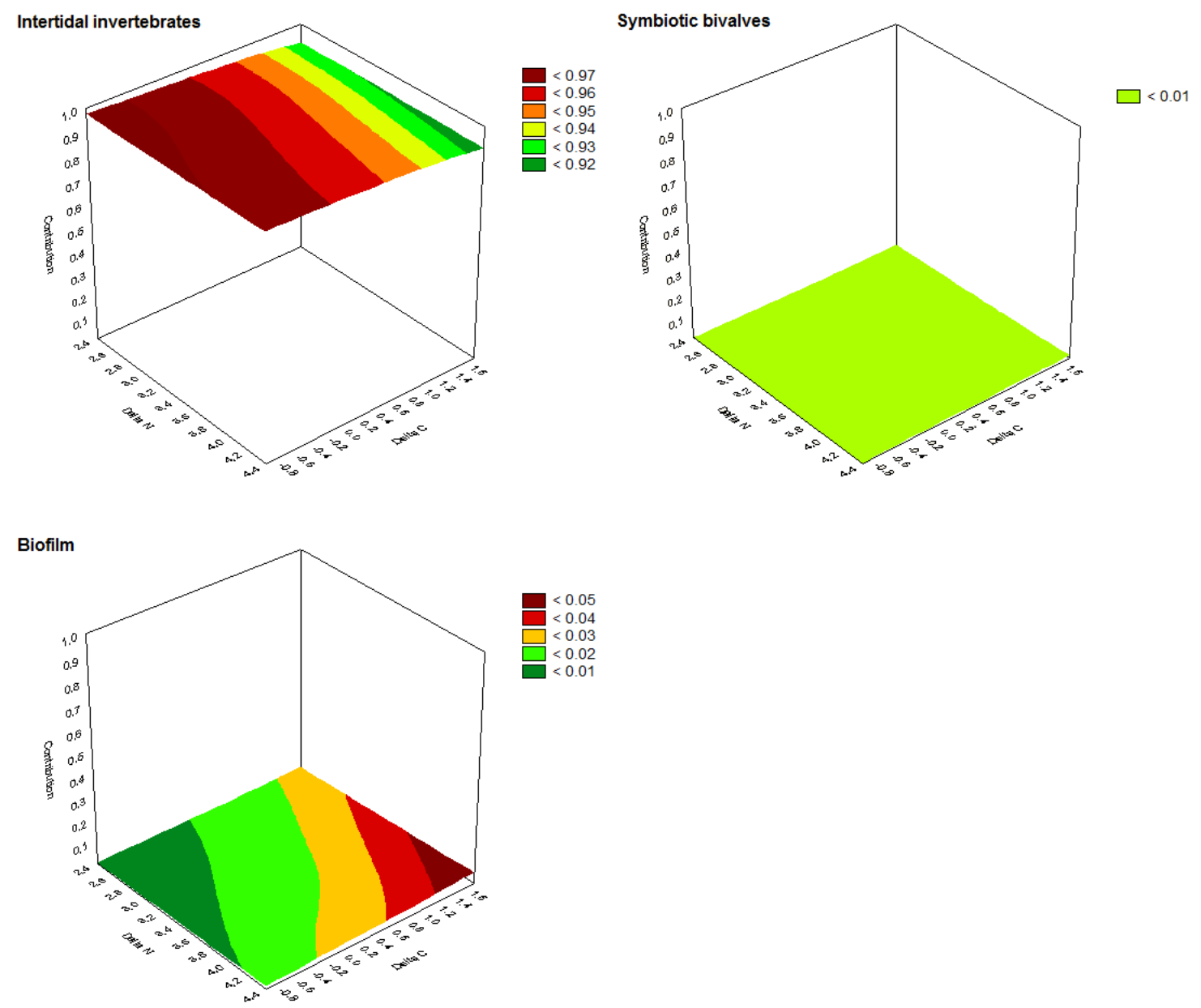
Red knot (Calidris canutus), Bijagós archipelago
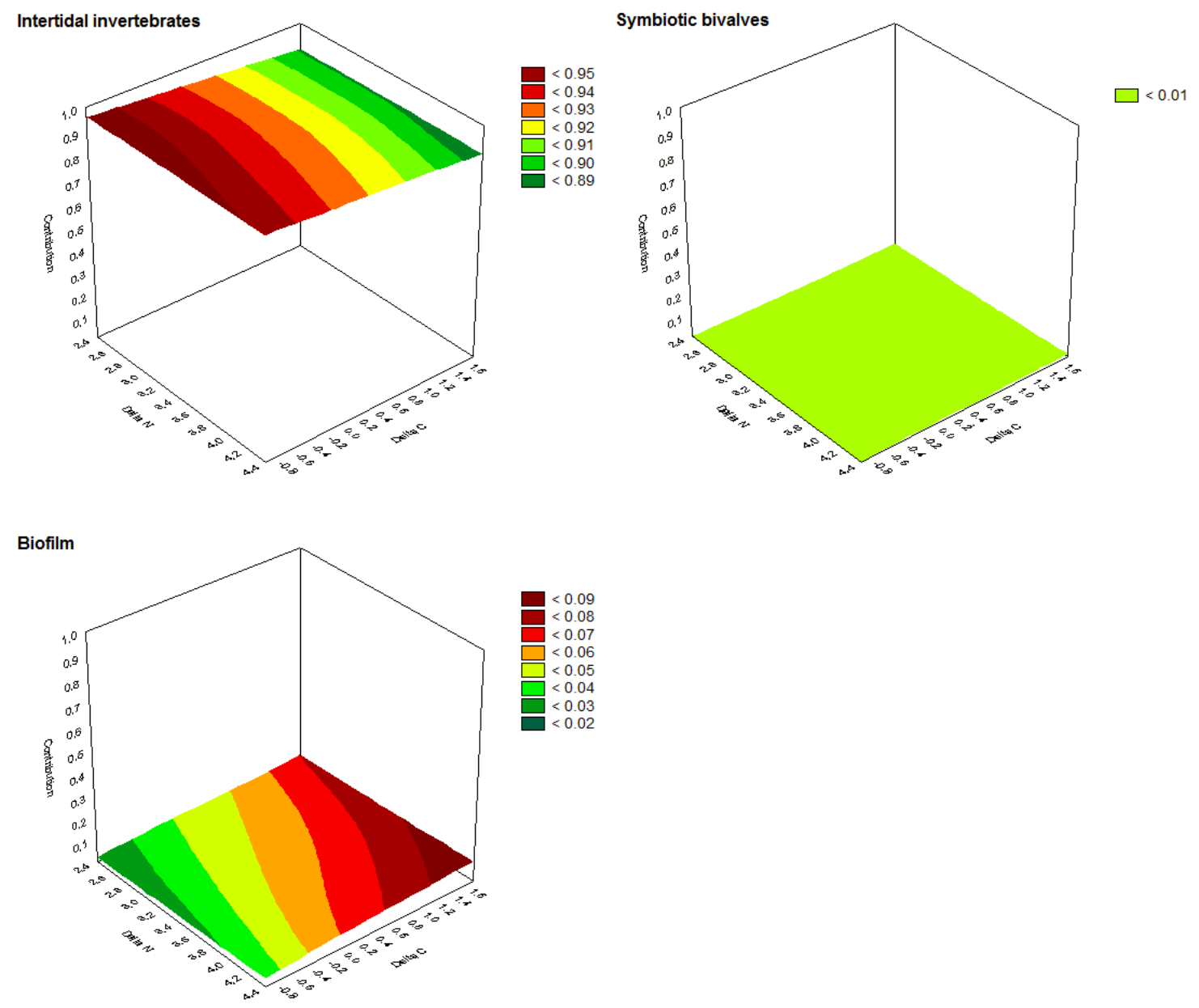


\section{Curlew sandpiper (Calidris ferruginea), Bijagós archipelago}
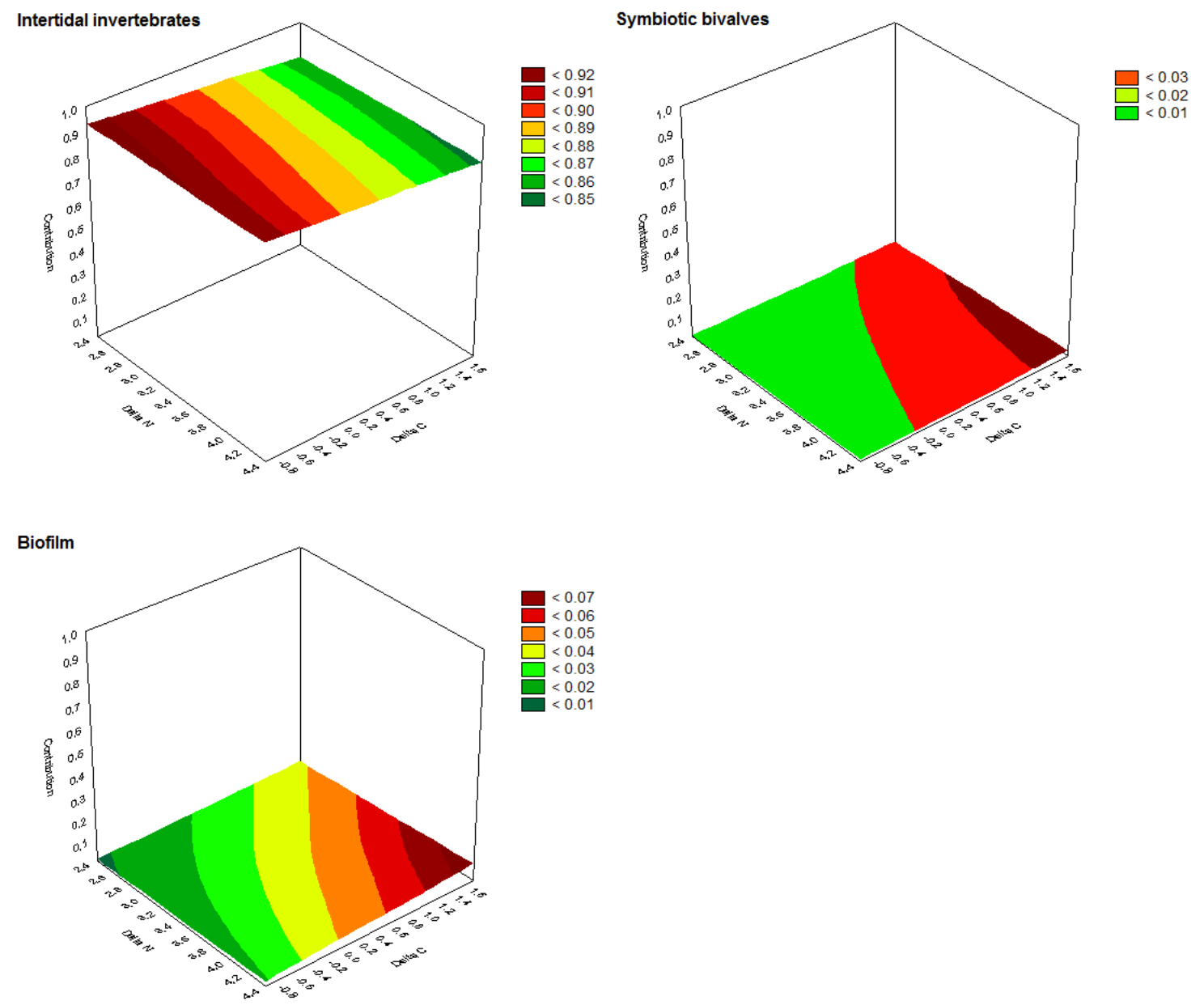
Ringed plover (Charadrius hiaticula), Bijagós archipelago
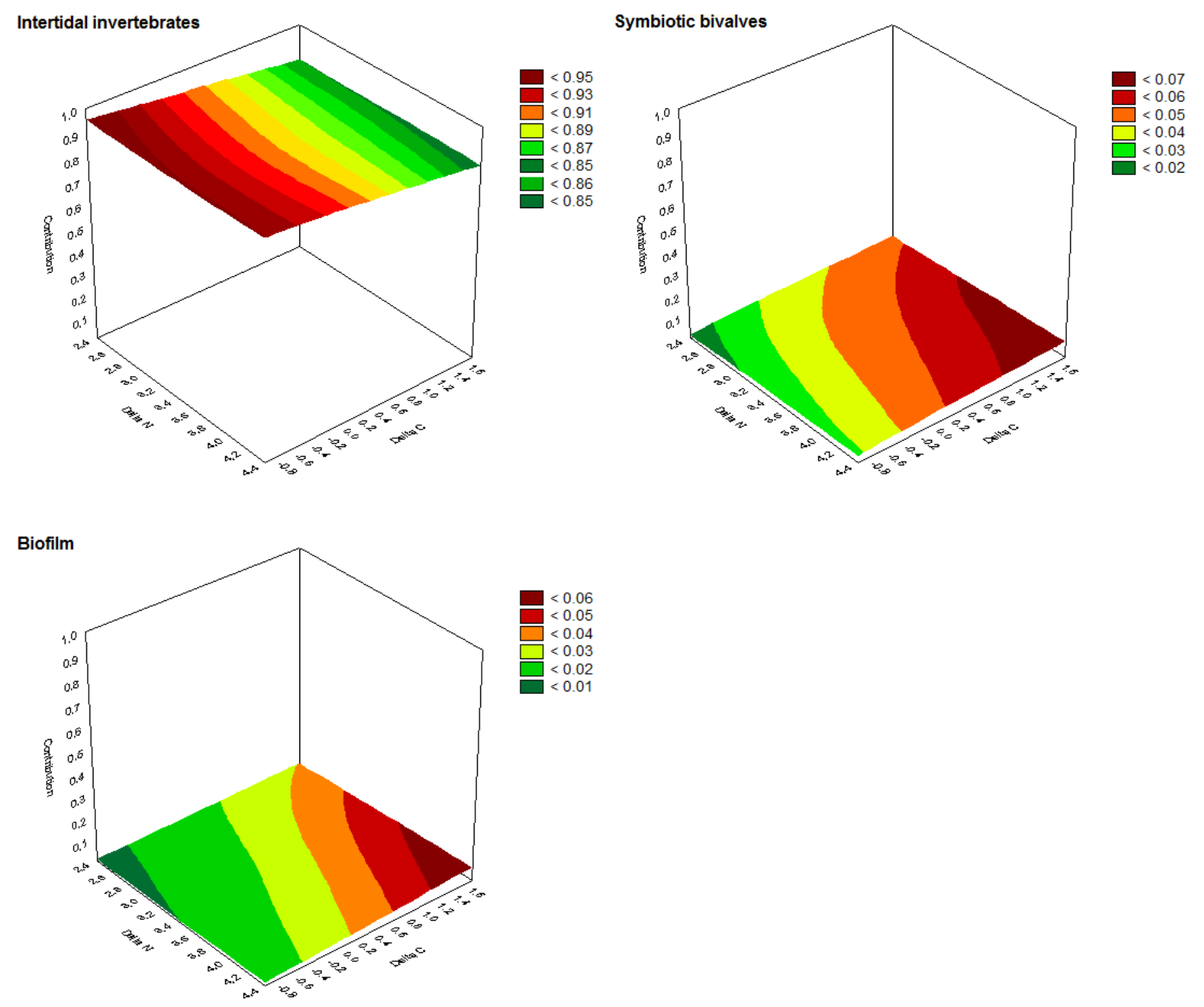
Bar-tailed godwit (Limosa lapponica), Bijagós archipelago
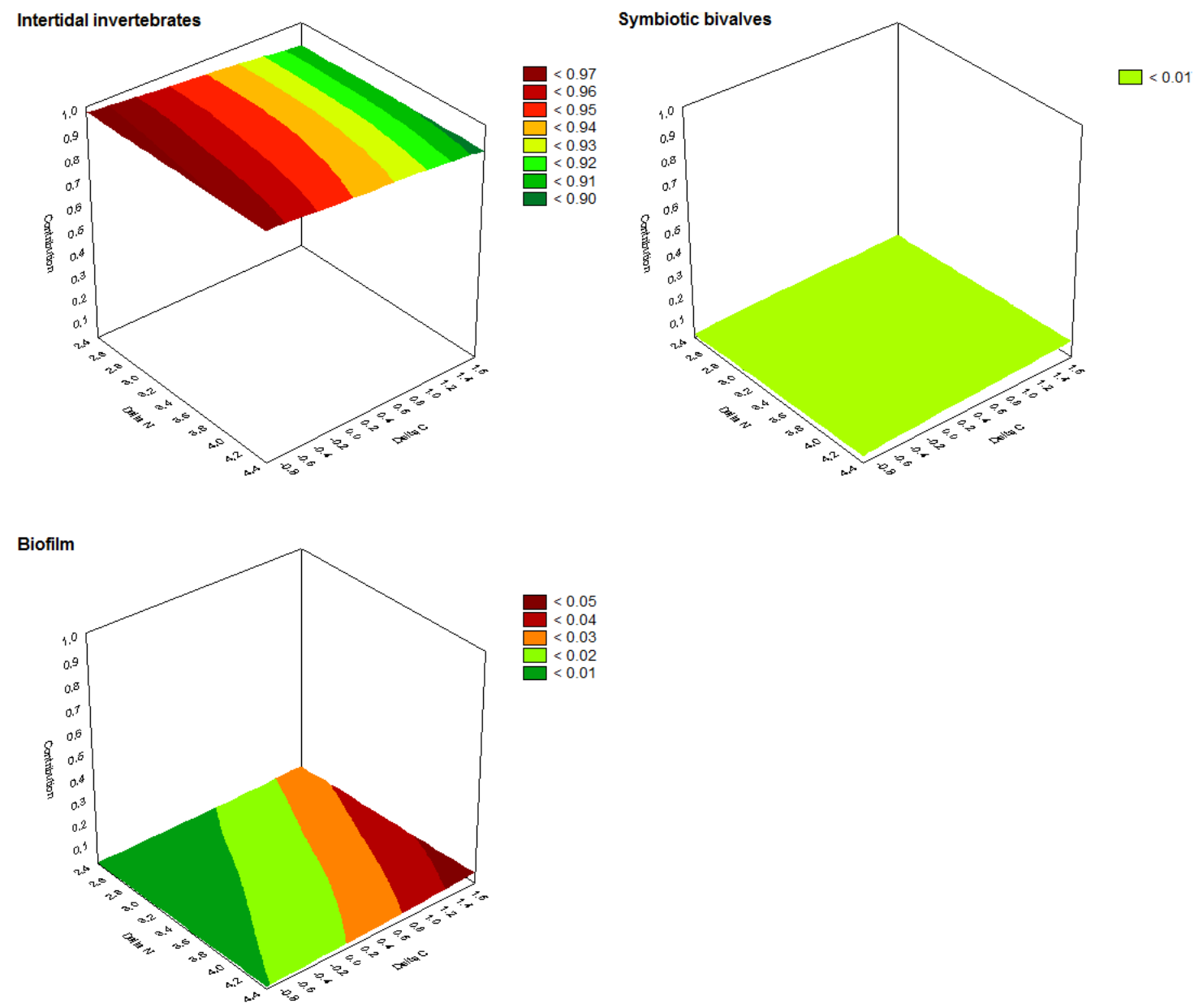
Grey plover (Pluvialis squatarola), Bijagós archipelago
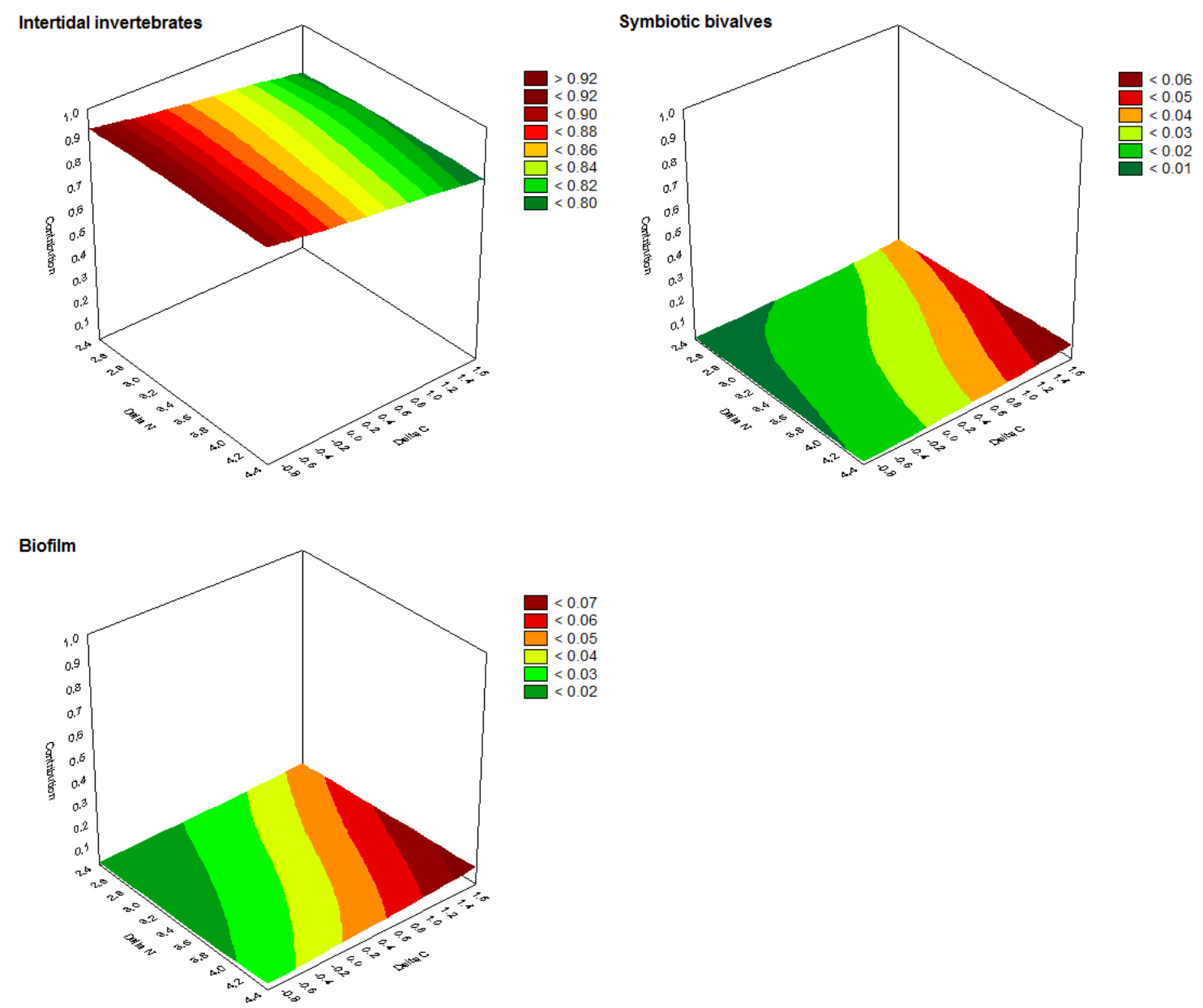
Redshank (Tringa totanus), Bijagós archipelago
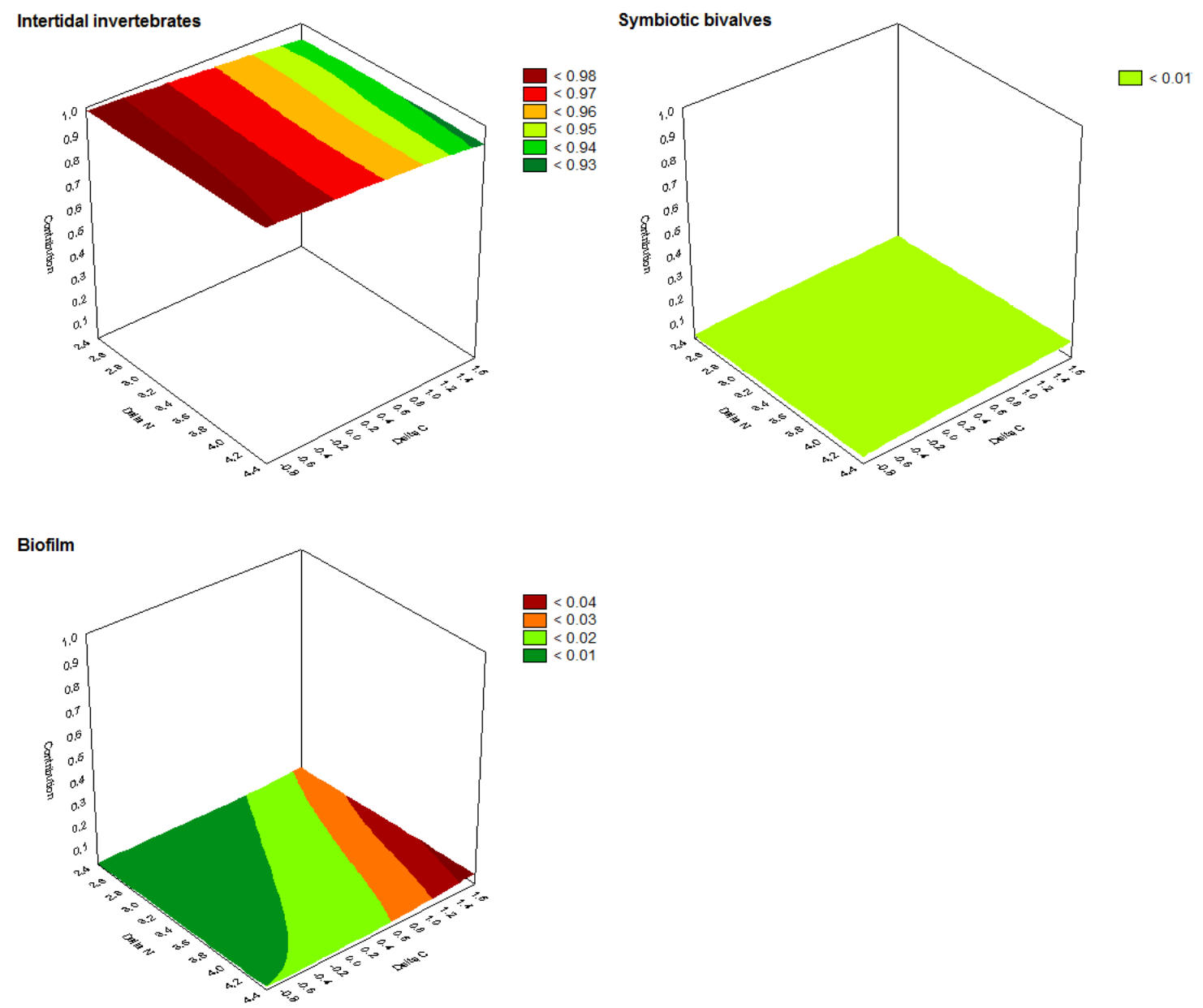
Table S1: $\delta 13 \mathrm{C}$ and $\delta 15 \mathrm{~N}$ values (mean $\pm \mathrm{SD}$ ) of potential food sources for shorebirds collected in the four study areas: Tejo estuary, Sidi-Moussa, Banc d'Arguin and Bijagós archipelago. $n=$ number of samples analysed followed by the number of individuals included in each sample. Potential food sources are: INTINV: intertidal macroinvertebrates, SALINV: saltpan macroinvertebrates, SYMCHE: intertidal bivalves with symbiotic chemoautotrophic bacteria, BIOFIL: biofilm, SEARHI: seagrass rhizomes.

\begin{tabular}{|c|c|c|c|c|}
\hline & $\delta^{15} \mathrm{~N}(\%)$ & $\delta^{13} \mathrm{C}(\%)$ & $\mathrm{n}$ & Food source \\
\hline \multicolumn{5}{|l|}{ Tejo estuary, Portugal } \\
\hline SOM & $8.05 \pm 0.54$ & $-20.82 \pm 0.62$ & 2 & BIOFIL \\
\hline Microalgae & $13.90 \pm 3.00$ & $-16.98 \pm 2.17$ & 2 & BIOFIL \\
\hline Scrobicularia plana & $15.87 \pm 0.85$ & $-16.13 \pm 1.18$ & $5(5-25)$ & INTINV \\
\hline Hydrobia ulvae & $15.26 \pm 0.54$ & $-13.15 \pm 0.36$ & $6(>25)$ & INTINV \\
\hline Hediste diversicolor & $15.70 \pm 1.21$ & $-13.44 \pm 1.32$ & $6(4-10)$ & INTINV \\
\hline Chironomidae & $9.51 \pm 1.33$ & $-17.73 \pm 0.29$ & $3(>20)$ & SALINV \\
\hline \multicolumn{5}{|l|}{ Sidi-Moussa, Morocco } \\
\hline SOM & 6.36 & -22.42 & 1 & BIOFIL \\
\hline Microalgae & 7.40 & -19.14 & 1 & BIOFIL \\
\hline Scrobicularia plana & $7.91 \pm 0.28$ & $-15.59 \pm 0.75$ & $3(5-10)$ & INTINV \\
\hline Hydrobia ulvae & $11.79 \pm 0.65$ & $-16.71 \pm 0.27$ & $3(>25)$ & INTINV \\
\hline Hediste diversicolor & $12.20 \pm 1.35$ & $-16.98 \pm 1.17$ & $4(4-10)$ & INTINV \\
\hline Chironomidae & $4.39 \pm 0.16$ & $-18.96 \pm 0.12$ & $3(>20)$ & SALINV \\
\hline Zostera noltii & 4.90 & -6.52 & $1(10)$ & SEARHI \\
\hline \multicolumn{5}{|c|}{ Banc d'Arguin, Mauritania } \\
\hline SOM & 4.39 & -17.89 & 1 & BIOFIL \\
\hline Microalgae & 5.68 & -20.83 & 1 & BIOFIL \\
\hline Dosinia isocardia & $7.07 \pm 0.47$ & $-17.06 \pm 0.56$ & $4(10-20)$ & INTINV \\
\hline Hydrobia ulvae & $5.00 \pm 0.35$ & $-11.75 \pm 0.40$ & $3(>50)$ & INTINV \\
\hline Ideotea chelipes & $4.97 \pm 0.41$ & $-8.17 \pm 0.18$ & $2(5-10)$ & INTINV \\
\hline Nereis sp. & $9.24 \pm 4.38$ & $-13.53 \pm 4.84$ & $3(7-20)$ & INTINV \\
\hline Glycera sp. & $11.16 \pm 3.62$ & $-12.50 \pm 3.08$ & $3(2-5)$ & INTINV \\
\hline Loripes lucinalis & $1.05 \pm 0.81$ & $-25.00 \pm 0.48$ & $9(10-20)$ & SYMCHE \\
\hline Zostera noltii & $0.82 \pm 0.72$ & $-8.15 \pm 2.17$ & $2(10)$ & SEARHI \\
\hline \multicolumn{5}{|c|}{ Bijagós archipelago, Guinea-Bissau } \\
\hline SOM & $6.52 \pm 1.19$ & $-19.04 \pm 0.97$ & 2 & BIOFIL \\
\hline Microalgae & 9.89 & -16.69 & 1 & BIOFIL \\
\hline Dosinia isocardia & $9.22 \pm 0.41$ & $-16.91 \pm 0.45$ & $3(5-10)$ & INTINV \\
\hline Tagelus adansonii & $9.29 \pm 0.11$ & $-15.90 \pm 0.22$ & $3(5-10)$ & INTINV \\
\hline Uca tangeri & $6.23 \pm 0.29$ & $-12.86 \pm 0.42$ & $3(2-5)$ & INTINV \\
\hline Eunicidae & $9.85 \pm 0.39$ & $-12.07 \pm 0.62$ & $2(2-5)$ & INTINV \\
\hline Glycera sp. & $10.23 \pm 0.77$ & $-16.72 \pm 1.36$ & $2(2-5)$ & INTINV \\
\hline Lucinidae & $-0.45 \pm 0.22$ & $-25.30 \pm 0.34$ & $3(5-10)$ & SYMCHE \\
\hline
\end{tabular}


Table S2: $\delta^{13} \mathrm{C}$ and $\delta^{15} \mathrm{~N}$ values (mean \pm SD) measured in nail samples of shorebird species in the four study areas: Tejo estuary, Sidi-Moussa, Banc d'Arguin and Bijagós archipelago. $n$ $=$ number of individuals.

\begin{tabular}{|c|c|c|c|}
\hline & $\delta^{15} \mathrm{~N}(\%)$ & $\delta^{13} \mathrm{C}(\%)$ & $\mathrm{n}$ \\
\hline \multicolumn{4}{|l|}{ Tejo estuary, Portugal } \\
\hline Arenaria interpres & $19.33 \pm 1.42$ & $-14.83 \pm 0.94$ & 10 \\
\hline Calidris alba & $19.94 \pm 0.32$ & $-15.85 \pm 0.32$ & 7 \\
\hline Calidris alpina & $17.29 \pm 2.41$ & $-15.73 \pm 1.88$ & 41 \\
\hline Calidris canutus & $15.02 \pm 2.03$ & $-16.21 \pm 1.74$ & 17 \\
\hline Calidris minuta & $14.43 \pm 1.76$ & $-16.54 \pm 1.36$ & 5 \\
\hline Charadrius hiaticula & $18.61 \pm 1.15$ & $-15.12 \pm 0.79$ & 15 \\
\hline Limosa limosa & $14.90 \pm 3.21$ & $-15.43 \pm 1.65$ & 10 \\
\hline Pluvialis squatarola & $18.70 \pm 1.39$ & $-14.30 \pm 0.88$ & 10 \\
\hline Tringa totanus & $19.41 \pm 1.13$ & $-15.10 \pm 0.95$ & 16 \\
\hline \multicolumn{4}{|l|}{ Sidi-Moussa, Morocco } \\
\hline Calidris alba & $11.65 \pm 0.90$ & $-17.07 \pm 0.92$ & 10 \\
\hline Calidris alpina & $11.66 \pm 1.58$ & $-13.79 \pm 1.07$ & 14 \\
\hline Calidris minuta & $10.26 \pm 1.37$ & $-18.56 \pm 1.63$ & 10 \\
\hline Charadrius hiaticula & $11.58 \pm 1.03$ & $-17.58 \pm 2.78$ & 10 \\
\hline Pluvialis squatarola & $10.95 \pm 2.10$ & $-17.56 \pm 2.08$ & 6 \\
\hline Tringa totanus & $9.91 \pm 0.60$ & $-16.64 \pm 1.85$ & 16 \\
\hline \multicolumn{4}{|c|}{ Banc d'Arguin, Mauritania } \\
\hline Arenaria interpres & $10.26 \pm 0.66$ & $-8.91 \pm 1.85$ & 15 \\
\hline Calidris alba & $9.57 \pm 0.58$ & $-8.52 \pm 0.97$ & 15 \\
\hline Calidris alpina & $9.41 \pm 0.63$ & $-7.96 \pm 0.77$ & 13 \\
\hline Calidris canutus & $8.92 \pm 1.18$ & $-12.95 \pm 3.26$ & 10 \\
\hline Calidris ferruginea & $8.93 \pm 0.54$ & $-8.94 \pm 0.83$ & 10 \\
\hline Calidris minuta & $6.09 \pm 0.46$ & $-6.05 \pm 0.99$ & 6 \\
\hline Charadrius hiaticula & $8.88 \pm 0.33$ & $-8.96 \pm 0.63$ & 10 \\
\hline Pluvialis squatarola & $10.39 \pm 0.56$ & $-10.81 \pm 0.49$ & 5 \\
\hline Tringa totanus & $9.16 \pm 0.95$ & $-8.53 \pm 1.78$ & 10 \\
\hline \multicolumn{4}{|c|}{ Bijagós archipelago, Guinea-Bissau } \\
\hline Arenaria interpres & $15.25 \pm 0.63$ & $-12.79 \pm 0.79$ & 8 \\
\hline Calidris alba & $13.81 \pm 0.90$ & $-11.68 \pm 0.77$ & 10 \\
\hline Calidris canutus & $13.15 \pm 1.02$ & $-12.80 \pm 1.47$ & 15 \\
\hline Calidris ferruginea & $12.57 \pm 0.80$ & $-11.20 \pm 1.03$ & 5 \\
\hline Charadrius hiaticula & $11.49 \pm 1.49$ & $-12.80 \pm 1.37$ & 16 \\
\hline Limosa lapponica & $14.56 \pm 1.44$ & $-12.21 \pm 0.47$ & 7 \\
\hline Pluvialis squatarola & $11.91 \pm 1.15$ & $-11.89 \pm 1.47$ & 8 \\
\hline Tringa totanus & $14.11 \pm 0.90$ & $-11.35 \pm 1.11$ & 10 \\
\hline
\end{tabular}


Table S3: Diet composition (proportion contribution of food sources in the diet) of shorebirds at the four study areas as estimated by stable isotope mixing models. In each case we present the median and the 0.025 and 0.975 quantiles of the stable isotope mixing models results. INTINV: intertidal invertebrates, SALINV: saltpan invertebrates, SYMCHE: intertidal bivalves with symbiotic chemoautotrophic bacteria, BIOFIL: biofilm, SEARHI: seagrass rhizomes.

\begin{tabular}{|c|c|c|c|c|c|}
\hline \multirow{3}{*}{ Tejo estuary, Portugal } & \multicolumn{5}{|c|}{ Percentage of diet } \\
\hline & INTINV & \multirow[t]{2}{*}{ SALINV } & SYMCHE & \multirow[t]{2}{*}{ BIOFIL } & \multirow[t]{2}{*}{ SEARHI } \\
\hline & & & & & \\
\hline Arenaria interpres & $\begin{array}{c}89.3 \\
(68.6-98.7)\end{array}$ & $\begin{array}{c}1.0 \\
(-0.2-21.1)\end{array}$ & & $\begin{array}{c}0.8 \\
(-0.1-12.9)\end{array}$ & \\
\hline Calidris alba & $\begin{array}{c}92.2 \\
(68.3-98.6)\end{array}$ & $\begin{array}{c}1.1 \\
(-0.3-22.7)\end{array}$ & & $\begin{array}{c}1.8 \\
(-0.7-18.5)\end{array}$ & \\
\hline Calidris alpina & $\begin{array}{c}74.2 \\
(64.3-84.1)\end{array}$ & $\begin{array}{c}14.5 \\
(0.7-32.3)\end{array}$ & & $\begin{array}{c}11.2 \\
(2.2-22.1)\end{array}$ & \\
\hline Calidris canutus & $\begin{array}{c}23.2 \\
(8.5-39.1)\end{array}$ & $\begin{array}{c}59.2 \\
(34.0-84.5)\end{array}$ & & $\begin{array}{c}17.6 \\
-0.7-31.6)\end{array}$ & \\
\hline Calidris minuta & $\begin{array}{c}34.4 \\
(12.5-52.3)\end{array}$ & $\begin{array}{c}30.4 \\
(9.5-56.0)\end{array}$ & & $\begin{array}{c}42.3 \\
(15.6-64.3)\end{array}$ & \\
\hline Charadrius hiaticula & $\begin{array}{c}85.9 \\
(73.6-95.0)\end{array}$ & $\begin{array}{c}4.2 \\
(-0.8-19.1)\end{array}$ & & $\begin{array}{c}0.4 \\
(-0.2-15.5)\end{array}$ & \\
\hline Limosa limosa & $\begin{array}{c}48.1 \\
(27.4-65.8)\end{array}$ & $\begin{array}{c}36.9 \\
(8.7-59.9)\end{array}$ & & $\begin{array}{c}3.3 \\
(-1.1-27.2)\end{array}$ & \\
\hline Pluvialis squatarola & $\begin{array}{c}87.2 \\
(74.0-97.3)\end{array}$ & $\begin{array}{c}3.3 \\
(-0.5-19.5)\end{array}$ & & $\begin{array}{c}1.4 \\
(-0.3-9.4)\end{array}$ & \\
\hline Tringa totanus & $\begin{array}{c}93.9 \\
(78.9-99.3)\end{array}$ & $\begin{array}{c}0.8 \\
(-0.2-14.1)\end{array}$ & & $\begin{array}{c}0.7 \\
(-0.1-12.4)\end{array}$ & \\
\hline \multicolumn{6}{|l|}{ Sidi-Moussa, Morocco } \\
\hline Calidris alba & $\begin{array}{c}45.1 \\
(33.3-58.4)\end{array}$ & $\begin{array}{c}42.5 \\
(24.3-64.2)\end{array}$ & & $\begin{array}{c}2.7 \\
(-0.2-21.2)\end{array}$ & $\begin{array}{c}9.2 \\
(2.0-16.8)\end{array}$ \\
\hline Calidris alpina & $\begin{array}{c}49.5 \\
(31.1-63.5)\end{array}$ & $\begin{array}{c}29.9 \\
(23.3-36.9)\end{array}$ & & $\begin{array}{c}3.2 \\
(-0.3-21.3)\end{array}$ & $\begin{array}{c}11.3 \\
(-0.4-26.6)\end{array}$ \\
\hline Calidris minuta & $\begin{array}{c}39.6 \\
(20.4-57.1)\end{array}$ & $\begin{array}{c}13.1 \\
(-1.7-33.9)\end{array}$ & & $\begin{array}{c}40.0 \\
(21.5-63.2)\end{array}$ & $\begin{array}{c}1.3 \\
(-0.2-11.3)\end{array}$ \\
\hline Charadrius hiaticula & $\begin{array}{c}52.6 \\
(38.1-65.3)\end{array}$ & $\begin{array}{c}41.4 \\
(21.3-58.0)\end{array}$ & & $\begin{array}{c}1.5 \\
(-0.5-17.3)\end{array}$ & $\begin{array}{c}6.7 \\
(-0.1-14.1)\end{array}$ \\
\hline Pluvialis squatarola & $\begin{array}{c}64.3 \\
(42.3-87.7)\end{array}$ & $\begin{array}{c}26.5 \\
(10.5-47.0)\end{array}$ & & $\begin{array}{c}2.6 \\
(-0.5-17.1)\end{array}$ & $\begin{array}{c}9.1 \\
(-1.2-19.8)\end{array}$ \\
\hline Tringa totanus & $\begin{array}{c}33.8 \\
(20.9-45.9)\end{array}$ & $\begin{array}{c}45.9 \\
(24.0-67.8)\end{array}$ & & $\begin{array}{c}1.2 \\
(-0.8-18.5)\end{array}$ & $\begin{array}{c}17.3 \\
(6.7-28.4)\end{array}$ \\
\hline \multicolumn{6}{|c|}{ Banc d'Arguin, Mauritania } \\
\hline Arenaria interpres & $\begin{array}{c}88.9 \\
(75.7-98.3)\end{array}$ & & $\begin{array}{c}0.2 \\
(-0.4-4.9)\end{array}$ & $\begin{array}{c}0.8 \\
(-0.1-8.1)\end{array}$ & $\begin{array}{c}5.7 \\
(0.9-19.1)\end{array}$ \\
\hline Calidris alba & $\begin{array}{c}80.4 \\
(65.9-93.7)\end{array}$ & & $\begin{array}{c}0.1 \\
(-0.1-3.9)\end{array}$ & $\begin{array}{c}0.9 \\
(-0.1-9.2)\end{array}$ & $\begin{array}{c}15.2 \\
(1.9-30.4)\end{array}$ \\
\hline Calidris alpina & $\begin{array}{c}78.7 \\
(61.6-94.8)\end{array}$ & & $\begin{array}{c}0.2 \\
(-0.1-5.5)\end{array}$ & $\begin{array}{c}0.9 \\
(-0.2-12.5)\end{array}$ & $\begin{array}{c}16.5 \\
(3.3-33.9)\end{array}$ \\
\hline Calidris canutus & $\begin{array}{c}57.1 \\
(39.6-77.6)\end{array}$ & & $\begin{array}{c}26.4 \\
(1.4-46.1)\end{array}$ & $\begin{array}{c}1.1 \\
(-0.1-14.4)\end{array}$ & $\begin{array}{c}10.1 \\
(2.9-25.2)\end{array}$ \\
\hline Calidris minuta & $\begin{array}{c}58.1 \\
(39.6-79.4)\end{array}$ & & $\begin{array}{c}0.9 \\
(-0.3-9.9)\end{array}$ & $\begin{array}{c}11.7 \\
(-0.6-29.4)\end{array}$ & $\begin{array}{c}28.4 \\
(3.8-50.3)\end{array}$ \\
\hline Calidris ferruginea & $\begin{array}{c}76.8 \\
(62.3-91.4)\end{array}$ & & $\begin{array}{c}0.6 \\
(-0.1-5.6)\end{array}$ & $\begin{array}{c}1.1 \\
(-0.1-8.2)\end{array}$ & $\begin{array}{c}16.5 \\
(1.7-31.4)\end{array}$ \\
\hline Charadrius hiaticula & $\begin{array}{c}81.3 \\
(63.4-99.2)\end{array}$ & & $\begin{array}{c}0.2 \\
(-0.1-5.8)\end{array}$ & $\begin{array}{c}0.7 \\
(-0.2-7.6)\end{array}$ & $\begin{array}{c}10.6 \\
(2.1-30.5)\end{array}$ \\
\hline Pluvialis squatarola & $\begin{array}{c}81.0 \\
(43.3-99.2)\end{array}$ & & $\begin{array}{c}1.0 \\
(-0.1-3.6)\end{array}$ & $\begin{array}{c}3.4 \\
(-0.4-12.0)\end{array}$ & $\begin{array}{c}10.8 \\
(1.2-28.8)\end{array}$ \\
\hline Tringa totanus & $\begin{array}{c}81.4 \\
(62.1-97.9)\end{array}$ & & $\begin{array}{c}0.8 \\
(-0.1-8.2)\end{array}$ & $\begin{array}{c}0.9 \\
(-0.2-15.3)\end{array}$ & $\begin{array}{c}10.6 \\
(1.9-28.6)\end{array}$ \\
\hline
\end{tabular}




\section{Bijagós archipelago, Guinea-Bissau}

Arenaria interpres

Calidris alba

Calidris canutus

Calidris ferruginea

Charadrius hiaticula

Limosa lapponica

Pluvialis squatarola

Tringa totanus
94.2

$(77.3-100.1)$

95.6

$(79.8-99.8)$

92.7

$(77.5-98.9)$

88.8

(61.1-98.9)

89.2

$(65.4-99.6)$

94.6

(80.0-99.9)

85.3

(54.4-96.9)

96.2

(81.4-99.8)
0.7

$(-0.1-5.8)$

0.3

0.7

$(-0.1-4.8) \quad(-0.2-15.3)$

$0.6 \quad 5.2$

$(-0.1-5.6) \quad(-1.6-18.4)$

$1.0 \quad 3.8$

$(-0.1-8.3) \quad(-1.5-25.3)$

$4.5 \quad 1.7$

$(-0.2-11.3) \quad(-1.0-20.4)$

$0.3 \quad 1.0$

$(-0.1-6.2) \quad(-0.2-16.8)$

$2.3 \quad 2.8$

$(-0.2-12.3) \quad(-0.4-29.8)$

$0.2 \quad 1.0$

$(-0.1-4.4) \quad(-0.1-11.5)$ 